IZA DP No. 9341

The Role of Social Networks in Cultural Assimilation

Thierry Verdier

Yves Zenou

September 2015

Forschungsinstitut zur Zukunft der Arbeit Institute for the Study of Labor 


\title{
The Role of Social Networks in Cultural Assimilation
}

\author{
Thierry Verdier \\ Paris School of Economics \\ and CEPR \\ Yves Zenou \\ Stockholm University, \\ IFN, CEPR and IZA
}

\section{Discussion Paper No. 9341 \\ September 2015}

\author{
IZA \\ P.O. Box 7240 \\ 53072 Bonn \\ Germany \\ Phone: +49-228-3894-0 \\ Fax: +49-228-3894-180 \\ E-mail: iza@iza.org
}

\begin{abstract}
Any opinions expressed here are those of the author(s) and not those of IZA. Research published in this series may include views on policy, but the institute itself takes no institutional policy positions. The IZA research network is committed to the IZA Guiding Principles of Research Integrity.

The Institute for the Study of Labor (IZA) in Bonn is a local and virtual international research center and a place of communication between science, politics and business. IZA is an independent nonprofit organization supported by Deutsche Post Foundation. The center is associated with the University of Bonn and offers a stimulating research environment through its international network, workshops and conferences, data service, project support, research visits and doctoral program. IZA engages in (i) original and internationally competitive research in all fields of labor economics, (ii) development of policy concepts, and (iii) dissemination of research results and concepts to the interested public.
\end{abstract}

IZA Discussion Papers often represent preliminary work and are circulated to encourage discussion. Citation of such a paper should account for its provisional character. A revised version may be available directly from the author. 
IZA Discussion Paper No. 9341

September 2015

\section{ABSTRACT}

\section{The Role of Social Networks in Cultural Assimilation*}

We develop a model where, in the first stage, minority individuals have to decide whether or not they want to assimilate to the majority culture while, in the second stage, all individuals (both from the majority and the minority group) embedded in a network have to decide how much effort they exert in some activity (say education). We show that the more central minority agents are in the social network, the more they assimilate to the majority culture. We also show that denser networks tend to favor assimilation so that, for example, it is easier to assimilate in a complete network than in a star-shaped network. We show that the subgameperfect equilibrium is not optimal because there is not enough activity and assimilation. We then endogeneize the network and show under which condition the ethnic minorities either assimilate to or separated themselves from the majority group.

JEL Classification: D85, J15, Z13

Keywords: assimilation, majority individuals, ethnic minorities, network centrality, network formation

Corresponding author:

Yves Zenou

Stockholm University

Department of Economics

10691 Stockholm

Sweden

E-mail: yves.zenou@ne.su.se

\footnotetext{
*We would like to thank Marc Sommer for very helpful comments.
} 


\section{Introduction}

An intense political and intellectual debate is taking place in Europe around migration issues. Rather than being centered on the economic costs and benefits of such inflows, the debate has instead focused on the perceived costs and benefits of cultural diversity. The attention paid to this issue is relatively novel in Europe and does represent a departure from the long-standing debate which has tended to emphasize racial discrimination as the key explanation of ethnic disadvantage. This is well illustrated by the hot debate in Europe about the veiling among Muslim women. and the recent votes in Switzerland against the construction of Muslim mosques and against immigrants. Many European countries are concerned about the cultural integration and the (lack of) assimilation of immigrants, that is whether the basic norms and values of the majority society are adopted by existing minority groups. ${ }^{1}$ For example, recently, the European eastern countries such as Hungary or Poland have clearly expressed their reluctance to welcome Syrian refugees fleeing war, who are mostly Muslims, arguing that their integration and assimilation to the European culture will be very difficult.

The assimilation outcomes of second generation youths have also been hotly debated amongst scholars, especially in the United States (Alba et al., 2011; Haller et al., 2011) where the immigrant population's growth in recent decades has raised questions about whether and how their children, the second generation, will integrate into American society. Current perspectives on second generation integration have evolved and are varied. Some scholars adhere to the segmented assimilation framework in which the second generation will assimilate into different segments of American society based on structural barriers and prejudices (Portes and Rumbaut, 2001; Portes and Zhou, 1993). Other scholars believe that the outcome between immigrant and mainstream culture is less dichotomous, and that immigrants and the American mainstream will eventually coalesce as lifestyles and patterns gradually become similar over time (Alba and Nee, 2003). Different studies have shown different significant influences on the assimilation process for immigrants: the quality of immigrant cohorts (Borjas, 1985), country of origin (e.g. Beenstock et al., 2010; Borjas, 1987, 1992; Chiswick and Miller, 2011), ethnic concentration (e.g. Edin et al., 2003; Lazear, 1999) and personal English skill (e.g. Chiswick and Miller, 1995, 1996; Dustmann and Fabbri, 2003; McManus et al., 1983).

An often overlooked structural factor of assimilation of ethnic minorities is the role of immigrant networks in the assimilation process. This is what we study in this paper.

To be more precise, we develop a model where all individuals, native and minorities (or immigrants), are embedded in a network. The network we are interested in is the one that forms between

\footnotetext{
${ }^{1}$ For an overview of these issues in Europe, see Kahanec and Zimmermann (2011).
} 
immigrants and natives but also between immigrants in the host country. As a result, we are not modeling the migration process and the role of network in migration (for such a model, see, e.g. Giulietti et al., 2014). Instead, we examine the role of networks in the host country on assimilation choices and outcomes. In this network, immigrants and natives possess different positions in the network and have different productivities.

There are two stages. In the first stage, minority individuals (immigrants) have to decide whether or not they want to assimilate to the majority culture while, in the second stage, all individuals (both from the majority and the minority group) embedded in a network ${ }^{2}$ have to decide how much effort they exert in some activity (say education or work on a job). Here both minority and majority workers belong to the same network. In this network, links can represent social or working relationships between people. There is a trade off for minority workers. If they choose assimilation, they will be more productive (in terms of education or in terms of skills on the job) because they are more adapted to the social norms of the host country (they know better the language and the habits of the host country). However, they need to pay a fixed cost of assimilation because it is costly, for example, to learn a new language. On the other hand, if they choose not to assimilate, they do not need to pay this cost but end up with a lower productivity, which decreases their outcomes (for example, their education level or their wage). As a result, the incentives for an individual belonging to the minority group to assimilate and adopt the culture of the majority are then directly related to the expected gains and costs that such a strategy implies. We consider a model where efforts are strategic complements. If, for example, we think of education, then if someone that I'm linked to studies hard then I enjoy more utility to study hard myself. Similarly, if we think of productivity on the job, then if I'm linked to someone who works hard, then I enjoy more utility from working hard.

We show that the more central agents in the network tend to have higher productivity than the less central ones and thus, the ethnic minorities who are more central in the network tend to assimilate more than those who are less central because the gains of assimilation are higher. We also show that, when the strength of interactions in the network increases, social interactions become more valuable and, because it is costly not to assimilate (in terms of productivity), more people choose to assimilate. This highlights the fact that endogenous assimilation choices affect the contribution to equilibrium efforts. For example, when the cost of assimilation decreases, more agent choose to assimilate to the majority culture, which, in turn, increases social interactions in the network and thus equilibrium productive efforts and outcomes. Furthermore, we find that denser networks tend to favor assimilation so that, for example, it is easier to assimilate in a complete

\footnotetext{
${ }^{2}$ The economics of networks is a growing field. See Jackson (2008), Ioannides (2012), Jackson et al. (2015) and Jackson and Zenou (2015) for recent overviews.
} 
network than in a star-shaped network.

We also show that the subgame-perfect equilibrium is not optimal because there is not enough activity and assimilation. To restore the first best, we find that it is optimal for the planner to give higher effort subsidies (to all individuals) but lower assimilation subsidies (to minority individuals) to more central agents in the social network. In the final part of the paper, we consider different communities that are not linked to each other and show that bridging them is always good for assimilation and also for total welfare.

We then extend our model to include network formation and, instead of having a binary choice of assimilation, we have a more "continuous" definition of assimilation, which is endogenously determined by the percentage of friends from the majority group each minority worker has. In this framework, the cost of forming links differ between intra-ethnic and inter-ethnic relationships. Due to cultural or language barriers, it is indeed always more costly to interact with someone from the other community than from own community. We assume that the cost of inter-ethnic relationships of two individuals negatively depends on the rate of exposure of these two agents to the other community. Indeed, the more an ethnic minority is "exposed" to the majority culture (by having an increasing fraction of friends from the majority group), the easier it will be for her to communicate and interact with other persons from the majority group and the lower will be the interaction costs between them. Using the concept of pairwise stability for equilibrium networks, we give conditions under which the two communities fully interact with each other (totally integrated communities) and when they do not interact at all (totally separated communities). We also provide some conditions for specific networks to be pairwise stable.

The rest of the paper unfolds as follows. In the next section, we relate our model to the relevant literatures and highlight our contribution. In Section 3, we expose the basic model, derive the two stages and characterize the subgame-perfect Nash equilibrium of this game. In Section 4, we illustrate our results with two specific networks: the star-shaped and the complete network. Section 5 is devoted to the welfare analysis and the subsidy policies aiming at restoring the first best. In Section 6, we consider separated communities and analyze how bridging them affects the outcomes of the individuals. Section 7 is devoted to network formation and assimilation choices. Finally, Section 8 concludes.

\section{Related literature}

Cultural transmission There is a literature related to the assimilation of ethnic minorities and immigrants that has looked at the cultural transmission, in particular, on the transmission of ethnic identity from parents and peers to children. Indeed, based on some works on anthropology 
and sociology (see, in particular, Boyd and Richerson, 1985, Cavalli-Sforza and Feldman, 1981; Phinney, 1990), there is a literature in economics initiated by Bisin and Verdier $(2000,2001)^{3}$ arguing that the transmission of a particular trait (religion, ethnicity, identity, etc.) is the outcome of a socialization inside (parents) and outside the family (peers and role models). Parents directly make various socialization choices, e.g., the rules and beliefs the family conforms to and how much time they spend with their children. This clearly affects the assimilation process of immigrants.

Ethnic identity The role of parents in the socialization of their own children is nonetheless limited by the children's pro-active role in choosing who to imitate and learn from, thereby directly shaping their own cultural identity. This is what Akerlof and Kranton (2010) have studied by developing the concept of identity economics, which aims to incorporate into economic theory the fact that people do not only pursue economic objectives, but also exert effort to gain and/or retain acceptance into a social group with which they identify. Many other papers have investigated the role of identity in the assimilation patterns of ethnic minorities (see, e.g. Bisin et al., 2011a,b; Panebianco, 2014; Verdier and Zenou, 2015). In a series of papers, Zimmermann et al. (2007), Constant and Zimmermann (2008), Constant et al. (2009) have proposed a new measure of the ethnic identity of migrants by modeling its determinants and explores its explanatory power for various types of their economic performance. They have proposed the ethnosizer, a measure of the intensity of a person's ethnic identity, which is constructed from information on language, culture, societal interaction, history of migration, and ethnic self-identification.

There is also an important literature that studies the concept of oppositional cultures among ethnic minorities. In this literature, ethnic groups may "choose" to adopt what are termed "oppositional" identities, that is, some actively reject the dominant ethnic (e.g., white) behavioral norms (they are oppositional) while others totally assimilate to it (see, in particular, Ainsworth-Darnell and Downey, 1998). Studies in the US and in Europe have found, for example, that African American students in poor areas may be ambivalent about learning standard English and performing well at school because this may be regarded as "acting white" and adopting mainstream identities (Fordham and Ogbu, 1986; Wilson, 1987; Delpit, 1995; Ogbu, 1997; Bisin et al., 2011b; Battu and Zenou, 2010; Fryer and Torelli, 2010; Patacchini and Zenou, 2016). On the theoretical side, Akerlof (1997), Austen-Smith and Fryer (2005), Selod and Zenou (2006), Battu et al. (2007), Bisin et al. (2011a) and De Marti and Zenou (2015) have proposed different models analyzing how oppositional identities affect the outcomes of ethnic minorities.

Compared to these two literatures, our main contribution is to put forward the role of social networks in the choice of assimilation of ethnic minorities and immigrants.

\footnotetext{
${ }^{3}$ For a review, see Bisin and Verdier (2011).
} 
Networks and assimilation There is a very small literature that looks at the impact of social networks on the assimilation choices of immigrants and ethnic minorities. Zhou and Bankston (1998) suggest that participating in ethnic religious institutions promotes upward assimilation through instilling an ethnic identity onto youths. Nguyen Le (2010) studies how do peer networks formed at youth groups affect assimilation trajectory for Vietnamese Americans. He shows that participation in Buddhist youth groups instills a Vietnamese-American identity on youths in Seattle and, in turn, this ethnic identity can lead to upward assimilation only if the individual is part of a peer network that promotes normative values. Peer networks formed at youth group shape individual behaviors because the peer networks' ties are remarkably strong. Members can relate to each other better than they can relate to their family members and school friends, since they share life experiences common amongst second generation Vietnamese Americans. Gang and Zimmermann (2000) show that ethnic network size has a positive effect on educational attainment, and a clear pattern is exhibited between countries-of-origin and education even in the second generation. Using the 2000 U.S. Census, Furtado and Theodoropoulos (2010) examine whether having access to native networks, as measured by marriage to a native, increases the probability of immigrant employment. They show that, indeed, marriage to a native increases immigrant employment rates. They present several pieces of evidence suggesting that networks obtained through marriage play an important part in explaining the relationship between marriage decisions and employment. Mouw et al. (2014) use a unique binational data on the social network connecting an immigrant sending community in Guanajuato, Mexico, to two destination areas in the United States. They test for the effect of respondents' positions in cross-border networks on their migration intentions and attitudes towards the United States using data on the opinions of their peers, their participation in cross-border and local communication networks. They find evidence of network clustering consistent with peer effects. Using German data and assuming that assimilation is taking place in the acquisition of education in Australia, Maani et al. (2015) show the ethnic capital network effects on immigrants' earnings assimilation are significant so that immigrants in Australia benefit from being spatially concentrated.

None of these studies, however, look at the exact network structure and the role of the position of the network in the assimilation process.

\section{The model}

There are $q+n$ individuals in the economy, $q$ individuals belonging to the majority group (type $W$ ) and $n$ individuals belonging to the minority group (type $B$ ). The network $g$ is a set of individuals $Q+N$, where $Q=\{1, \ldots, q\}, q \geq 2$ (set of majority individuals) while $N=\{1, \ldots, n\}, n \geq 2$ 
(set of minority individuals), and a set of links or direct connections between them. The adjacency matrix $\mathbf{G}=\left[g_{i j}\right]$ keeps track of the direct connections in the network. By definition, agents $i$ and $j$ are directly connected if and only if $g_{i j}=1$; otherwise, $g_{i j}=0$. We assume that if $g_{i j}=1$, then $g_{j i}=1$, so the network is undirected. By convention, $g_{i i}=0$.

The timing of the model is as follows. In the first stage, each ethnic minority $i$ decides whether she wants to keep her culture of origin $\left(\alpha=\alpha_{0}\right)$ or assimilate to the culture of the majority group of the host country $\left(\alpha=\alpha_{1}\right)$. Individuals from the majority group do not need to make this choice since, by definition, they are assimilated to their own culture (i.e. $\alpha=\alpha_{1}$ ). In the second stage, given that the position in the network is given, each individual (ethnic minorities and individuals from the majority group) has to decide how much effort to put in some activity, say education. We assume that more assimilated minorities (those who choose $\alpha_{1}$ ) are more productive (in terms of acquiring education or in terms of productivity on the job) than less assimilated minorities (those who choose $\alpha_{0}$ ) because the latter lack the exact skills required for jobs in the host country (for example, they do not speak fluently the language of the host country, they have different ways of working, etc.; see e.g. Lazear, 1999; De Marti and Zenou, 2015 and our discussion in Section 2). To be more precise, assimilated minority individuals have an education productivity of $\alpha_{1}=\alpha$ but have a cost of assimilation equal to $c$ while oppositional minority individuals have a productivity of $\alpha_{0}=\alpha-t$ but have no cost of assimilation. In other words, $c$ is the assimilation cost (because of peer pressure from the ethnic group or simply because it is costly to learn the new culture and language of the majority group) while $t$ is the productivity cost of being non-assimilated (because of poorer language skills that affect the productivity of education or on-the-job skill). We have $\alpha_{i} \in\{\alpha-t, \alpha\}, \forall i=1,2, \ldots, n$. As usual, we solve the model backwards.

\subsection{Second stage: Choosing education effort}

Let us solve the second stage where individuals choose simultaneously their education efforts.

\subsubsection{Preferences}

The preferences of individual $i$ in network $g$ is given by:

$$
U_{i}\left(x_{i}, \mathbf{x}_{-\mathbf{i}}, g\right)=\alpha_{i} x_{i}-\frac{1}{2} x_{i}^{2}+\theta \sum_{j=1}^{q+n} g_{i j} x_{i} x_{j}
$$

where $x_{i}$ is the education effort ${ }^{4}$ that individual $i$ exerts, $\mathbf{x}_{-\mathbf{i}}$ is the corresponding vector of efforts for the other $q+n-1$ agents in the network. The parameter $\theta>0$ measures the synergy of social

\footnotetext{
${ }^{4}$ We interpret our model in terms of education effort but, of course, it can be interpreted in terms of skill or productive effort.
} 
interactions between linked individuals. When $\theta$ is positive, an increase in $x_{j}$, the education effort of individual $j$ who is directly linked to $i$, increases the marginal utility for individual $i$ of exerting own effort $x_{i}$. For the majority group (type $W$ ), by definition, $\alpha_{i}=\alpha, \forall i=1,2, \ldots, q$, while, for the minority group (type $B$ ), $\alpha_{i}=\alpha$ for those who assimilate to the majority group while $\alpha_{i}=\alpha-t$ for those who do not assimilate (i.e. oppositional). We assume $\alpha>t$, so that $\alpha_{i}>0, \forall i=1,2, \ldots n$. The utility function (1) has two parts. The first one, $\alpha_{i} x_{i}-\frac{1}{2} x_{i}^{2}$, is the utility of exerting $x_{i}$ when there is no interaction with other individuals. The second part, $\theta \sum_{j=1}^{q+n} g_{i j} x_{i} x_{j}$, is what is obtained by interacting with direct friends in the network.

In the second stage, each agent $i$ chooses $x_{i}$ to maximize (1) taking the structure of the network and the effort choices of other agents as given. In Appendix 1, we define the Katz-Bonacich centrality, which will be useful in the characterization of the Nash equilibrium.

\subsubsection{Nash equilibrium in education efforts}

For each $i=1, \ldots, n$, the first-order condition with respect to $x_{i}$ is given by:

$$
x_{i}^{*}\left(\alpha_{i}, \boldsymbol{\alpha}_{-\mathbf{i}}, g\right)=\alpha_{i}+\theta \sum_{j=1}^{q+n} g_{i j} x_{j}^{*} \quad \forall i=1,2, \ldots n+q .
$$

The optimal education effort choice of agent $i$ is a linear function of the education efforts of the agents to whom $i$ is directly connected in the network. Solving for the $(q+n \times 1)$ vector $\mathbf{x}$ and using (29) gives the Nash equilibrium visit vector $\mathbf{x}^{*}$ :

$$
\mathbf{x}^{*}=(\mathbf{I}-\theta \mathbf{G})^{-1} \boldsymbol{\alpha}=\mathbf{M} \boldsymbol{\alpha}
$$

where $\mathbf{M} \equiv(\mathbf{I}-\theta \mathbf{G})^{-1}$ is a $(q+n \times q+n)$ matrix. Denote by $\rho(\mathbf{G})$ the spectral radius of the adjacency matrix $\mathbf{G}$. Then, we have the following result:

Proposition 1 (Equilibrium efforts) If $\theta \rho(\mathbf{G})<1$, there exists a unique, interior Nash equilibrium in effort choices, which, for each individual $i$, is given by:

$$
x_{i}^{*}\left(\alpha_{i}, \boldsymbol{\alpha}_{-\mathbf{i}}, g\right)=b_{\alpha_{i}}(g, \theta) .
$$

The proof of this proposition can be found in Ballester et al. (2006) and Calvó-Armengol et al. (2009). The Nash equilibrium number of efforts $x_{i}^{*}\left(\alpha_{i}, \boldsymbol{\alpha}_{-\mathbf{i}}, g\right)$ depends on the position in the social network and the assimilation choices of the minority workers. Proposition 1 implies that an agent who is more central in the social network, as measured by her Katz-Bonacich centrality, will make more (education) effort in equilibrium. Intuitively, agents who are better connected have more to gain from interacting with others and so exert higher effort for any vector of assimilation choices. It is straightforward to verify that $x_{i}^{*}\left(\alpha_{i}, \boldsymbol{\alpha}_{-\mathbf{i}}, g\right)$ increases with $\alpha$, the degree of assimilation, and $\theta$, and the intensity of social interactions but decreases with the productivity cost $t$. 


\subsection{First stage: Choosing the degree of assimilation}

As stated above, there is an exogenous cost differential $c>0$ associated with assimilation. We consider any network with $q+n$ agents but only focus on the choice of the ethnic minority population given by $N=\{1, \ldots, n\}$. We assume that the $q$ individuals from the majority group occupy the most central position (in terms of Katz-Bonacich centrality) in the network (for historical reasons and because of old-boy networks). In fact, this assumption is not necessary for the results of this section but will facilitate the characterization of equilibrium.

Agents from the minority group (type $B$ ) choose the $\alpha_{i}$ that maximizes their net utility, taking the efforts of all other agents (including the majority individuals) as given. We look at subgameperfect equilibria. We have seen in the previous section (Proposition 1) that, if $\theta \rho(\mathbf{G})<1$, there exists a unique effort level for each individual $i$ given by: $x_{i}^{*}\left(\alpha_{i}, \boldsymbol{\alpha}_{-\mathbf{i}}, g\right)=b_{\alpha_{i}}(g, \theta)$. Using the best-response function (2) and plugging it into (1), we can write the equilibrium utility level of individual $i$ as:

$$
U_{i}^{*}\left(x_{i}^{*}, \mathbf{x}_{-\mathbf{i}}^{*}, g\right)=\frac{1}{2}\left[x_{i}^{*}\left(\alpha_{i}, \boldsymbol{\alpha}_{-\mathbf{i}}, g\right)\right]^{2}=\frac{1}{2}\left[b_{\alpha_{i}}(g, \theta)\right]^{2}
$$

where $x_{i}^{*}\left(\alpha_{0}, \mathbf{x}_{-\mathbf{i}}, g\right)$ and $x_{i}^{*}\left(\alpha_{1}, \mathbf{x}_{-\mathbf{i}}, g\right)$ are the equilibrium effort of individual $i$ if she is oppositional or not assimilated $\left(\alpha_{i}^{*}=\alpha_{0}\right)$ and if she is assimilated $\left(\alpha_{i}^{*}=\alpha_{1}\right)$, respectively. We need now to solve the first stage of the game, i.e. the assimilation choices.

Define $\mathcal{A}$ as the set of assimilated individuals (i.e. all individuals from the minority group who choose $\alpha_{1}=\alpha$ and all individuals from the majority group) and $\mathcal{O}$ as the set of oppositional individuals (i.e. all minority individuals who choose $\alpha_{0}=\alpha-t$ ). If individual $i$ choose to assimilate $\left(\alpha_{1}=\alpha\right)$, her equilibrium utility is equal to:

$$
U_{i}^{*}\left(x_{i}^{*}\left(\alpha_{1}, \mathbf{x}_{-\mathbf{i}}, g\right), \mathbf{x}_{-\mathbf{i}}^{*}, g\right)=\frac{1}{2}\left[\sum_{j \in \mathcal{A}-\{i\}} \sum_{k=0}^{+\infty} \theta^{k} g_{i j}^{[k]} \alpha+\sum_{j \in \mathcal{O}-\{i\}} \sum_{k=0}^{+\infty} \theta^{k} g_{i j}^{[k]}(\alpha-t)+\sum_{k=0}^{+\infty} \theta^{k} g_{i i}^{[k]} \alpha\right]^{2}-c
$$

We have here decomposed the Katz-Bonacich centrality $b_{\alpha_{i}}(g, \theta)$ into self-loops $\left(m_{i i}=\sum_{k=0}^{+\infty} \theta^{k} g_{i i}^{[k]}\right)^{5}$ and non self-loops $\left(m_{i j}=\sum_{k=0}^{+\infty} \theta^{k} g_{i j}^{[k]}\right)$ and give different weights to these paths depending if agents are assimilated (weight $\alpha$ ) or oppositional (weight $\alpha-t$ ). If individual $i$ chooses to be oppositional $\left(\alpha_{0}=\alpha-t\right)$, her equilibrium utility is given by:

$$
U_{i}^{*}\left(x_{i}^{*}\left(\alpha_{0}, \mathbf{x}_{-\mathbf{i}}, g\right), \mathbf{x}_{-\mathbf{i}}^{*}, g\right)=\frac{1}{2}\left[\sum_{j \in \mathcal{A}-\{i\}} \sum_{k=0}^{+\infty} \theta^{k} g_{i j}^{[k]} \alpha+\sum_{j \in \mathcal{O}-\{i\}} \sum_{k=0}^{+\infty} \theta^{k} g_{i j}^{[k]}(\alpha-t)+\sum_{k=0}^{+\infty} \theta^{k} g_{i i}^{[k]}(\alpha-t)\right]^{2}
$$

Thus, individual $i$ will assimilate if and only if $U_{i}^{*}\left(x_{i}^{*}\left(\alpha_{1}, \mathbf{x}_{-\mathbf{i}}, g\right), \mathbf{x}_{-\mathbf{i}}^{*}, g\right)>U_{i}^{*}\left(x_{i}^{*}\left(\alpha_{0}, \mathbf{x}_{-\mathbf{i}}, g\right), \mathbf{x}_{-\mathbf{i}}^{*}, g\right)$.

\footnotetext{
${ }^{5}$ See Appendix 1 for the interpretations of the $m_{i i} \mathrm{~s}$ and $m_{i j} \mathrm{~s}$.
} 
We would now like to deal with the issues of existence and uniqueness of the subgame-perfect equilibrium assimilation-effort. Since all the $q$ individuals from the majority group are the most central agents (in terms of Katz-Bonacich centrality), we can rank them starting with agent 1, who is the most central agent from the majority group, $m_{11}=\max _{i} m_{i i}, \forall i \in Q$, then agent 2 , who has the next highest centrality, etc. until we reach agent $q$ who has the lowest centrality in the network among the majority individuals, i.e. $m_{q, q}=\min _{i} m_{i i}, \forall i \in Q$. Then, we start with the agent who has the highest centrality among the minority individuals, i.e. $m_{q+1, q+1}=\max _{i} m_{i i}, \forall i \in N$. Then, we have the second minority agent, who has the next highest centrality among the minority group, etc., until we reach agent $n$ who has the lowest centrality in the network among the majority individuals, i.e. $m_{q+n, q+n}=\min _{i} m_{i i}, \forall i \in N$. Define each minority individual by her type, where the type of an agent is her Katz-Bonacich centrality (or her $m_{i i}$ ). Since two minority agents can have the same centrality, there are $\omega \leq n$ types in each network of $q+n$ agents. Observe that $\omega=n$ if there are no minority individuals who have the same position (or centrality) in the network. For example, in a star network with 10 minority individuals (and no majority individuals), $\omega=2$ and $n=10$ while, in a complete network with the same number of individuals, $\omega=1$ and $n=10$. For the characterization of the subgame-perfect equilibrium, the types of the majority individuals do not matter as they are all assimilated.

To characterize the equilibria, we will use a technique similar to the one developed in Helsley and Zenou (2014). Denote by

$$
\Phi^{\mathcal{A}}\left(m_{i, i}\right) \equiv t(2 \alpha-t)\left(m_{i, i}\right)^{2}+2 t\left(\alpha \sum_{j=1, j \neq i}^{q+n} m_{i, j}\right) m_{i, i}, i=1, \ldots, n
$$

where all the $m_{i i}$ and $m_{i j}$ s are defined by the cells of the $(q+n \times q+n)$ matrix $\mathbf{M}=[\mathbf{I}-\theta \mathbf{G}]^{-1}$. In (6), $\Phi^{\mathcal{A}}\left(m_{i, i}\right)$ is the incentive function for a given ethnic individual $i \in N$ to choose to assimilate or not when all other minority (and, of course, majority) agents are assimilated. We have a first intuitive result:

Proposition 2 Assume $\theta \rho(\mathbf{G})<1$ and consider any network of $q+n$ agents with $\omega \leq n$ types for the minority individuals. In any equilibrium, two minority workers with the same Katz-Bonacich centrality make the same assimilation choice and agents with higher Katz-Bonacich centrality cannot be oppositional if agents with lower Katz-Bonacich centrality are assimilated. Moreover, the number of equilibria is equal to the number of types of minority individuals plus one, i.e. $\omega+1$.

Let us now derive our existence and uniqueness result: 
Proposition 3 (Existence and uniqueness of equilibrium assimilation behaviors) Assume $\theta \rho(\mathbf{G})<1$ and consider any network of $q+n$ agents with $\omega \leq n$ types for the minority individuals. If the number of types of minority individuals is the same as the number of minority individuals, we can characterize the subgame-perfect equilibria as follows:

(i) If

$$
2 c<\Phi^{\mathcal{A}}\left(m_{q+n, q+n}\right)
$$

there exists a unique Assimilation equilibrium where all agents choose to assimilate, i.e. $\mathcal{A}=$ $Q+N$ and $\mathcal{O}=\varnothing$.

(ii) If

$$
\Phi^{\mathcal{A}}\left(m_{q+n, q+n}\right)<2 c<\Phi^{\mathcal{A}}\left(m_{q+n-1, q+n-1}\right)-2 t^{2} m_{q+n-1, q+n} m_{q+n-1, q+n-1}
$$

there exists a unique Assimilation-Oppositional equilibrium such that $\mathcal{A}=Q+N-\{n\}$ and $\mathcal{O}=\{n\}$.

(iii) If

$$
\begin{aligned}
& \Phi^{\mathcal{A}}\left(m_{q+n-1, q+n-1}\right)-2 t^{2} m_{q+n-1, q+n} m_{q+n-1, q+n-1} \\
< & 2 c<\Phi^{\mathcal{A}}\left(m_{q+n-2, q+n-2}\right)-2 t^{2}\left(m_{q+n-2, q+n-1}+m_{q+n-2, q+n}\right) m_{q+n-2, q+n-2}
\end{aligned}
$$

there exists a unique Assimilation-Oppositional equilibrium such that $\mathcal{A}=Q+N-\{n-1, n\}$ and $\mathcal{O}=\{n-1, n\}$.

(iv) If

$$
\begin{aligned}
& \Phi^{\mathcal{A}}\left(m_{q+n-2, q+n-2}\right)-2 t^{2}\left(m_{q+n-2, q+n-1}+m_{q+n-2, q+n}\right) m_{q+n-2, q+n-2} \\
< & 2 c<\Phi^{\mathcal{A}}\left(m_{q+n-3, q+n-3}\right)-2 t^{2}\left(\sum_{j \in \mathcal{O}} m_{q+n-3, q+j}\right) m_{q+n-3, q+n-3}
\end{aligned}
$$

there exists a unique Assimilation-Oppositional equilibrium such that $\mathcal{A}=Q+N-\{n-2, n-$ $1, n\}$ and $\mathcal{O}=\{n-2, n-1, n\}$.

$(v)$ etc. until we arrive at agent 1 who has the highest centrality among the minority group. Then,

(vi) If

$$
\Phi^{\mathcal{A}}\left(m_{q+1, q+1}\right)-2 t^{2}\left(\sum_{j \in \mathcal{O}-\{1\}} m_{q+1, q+j}\right) m_{q+1, q+1}<2 c
$$

there exists a unique Oppositional equilibrium where all minority individuals choose not to assimilate, i.e. $\mathcal{A}=Q$ and $\mathcal{O}=N$. 
If the number of types is less than the number of minority individuals, then each step described above has to be made by type and not by individual so that each subscript refers to types and not to individuals.

This proposition characterizes the (subgame-perfect Nash) equilibrium assimilation choices and shows that there always exists a unique equilibrium within each interval. The key object is $\Phi^{\mathcal{A}}\left(m_{i i}\right)$, which is the "incentive function" when there is an Assimilated equilibrium, i.e. when all minority individuals choose to assimilate. Indeed, when, for all $i, \Phi^{\mathcal{A}}\left(m_{i i}\right)>2 c$, all minority individuals choose to assimilate because the cost of assimilation is low enough compared to the gain from assimilation in terms of productivity and synergies from other individuals (both from the majority and minority groups). In that case, there is a unique Assimilation equilibrium. Then, when we start to increase the cost of assimilation, $c$, and change the decision of minority workers from assimilation to oppositional, the weight in the Katz-Bonacich centrality changes from $\alpha$ (when assimilated) to $\alpha-t$ (when oppositional). This corresponds to the terms of both the right-hand side and lefthand side of each inequality since this is what is needed to be compensated for the agents who are oppositional compared to the Assimilation equilibrium where these agents are all assimilated. Observe that there cannot be multiple equilibria within the same set of parameters.

This result is related to the literature on ethnic identity highlighted in Section 2. In this literature, ethnic groups may "choose" to adopt "oppositional" identities, that is, some actively reject the dominant ethnic (e.g., white) behavioral norms (they are oppositional) while others totally assimilate to it. Proposition 3 shows that these choices, especially the assimilation choice, negatively depends on the cost $c$ of assimilating to the majority culture, positively on $t$, the productivity cost of being non-assimilated, and positively on the position (or centrality) of the individual in the network.

The impact of $c$ on assimilation choices is well-documented. Indeed, studying the assimilation of Muslims in France, Adida et al. (2014) show that if the cost of assimilation is sufficiently high (possibly due to taste-based discrimination by the dominant group), it then becomes rational for members of the minority to eschew assimilation. This is consistent with Proposition 3 but also with other theoretical models (see, in particular, Laitin, 1995; Akerlof and Kranton, 2010; Fang and Loury, 2005; Bisin et al., 2011a). Also, focusing on Mexican immigrants in the United States, Mouw et al. (2014) show that respondents who speak English poorly are more likely to report that they would be happier living in Mexico (a measure of assimilation). Finally, Dustmann (1996) documents that immigrants from more distant cultural backgrounds (e.g., Turks, Greeks) exhibit a lower assimilation tendency into German society. Language proficiency and education are other important determinants of cultural assimilation, as is labor market integration (Dustmann, 
1994, 1996). In these studies, discrimination, language skills, education and cultural distance are a measure of $c$, the cost of assimilating to the majority culture.

If we now consider the impact of $t$ on assimilation choices, there is also plenty of evidence. For example, Constant et al. (2009), Zimmerman (2007) and Constant and Zimmerman (2008) show that there are positive and significant impacts of assimilation with the host culture on economic behavior such as work probability, and earnings. In particular, Constant and Zimmermann (2009) find that assimilation with German culture significantly increases the probability of work for male immigrants, relative to those identified as separated or marginalized. More recently, Gorinas (2014), shows that immigrants who share social norms with the majority experience significantly better employment outcomes, particularly first-generation immigrant women. These results are also consistent with Proposition 3 where $t$ can capture workers' productivity but also the probability of finding a job.

Finally, the most original result in Proposition 3 is the role of social networks, in particular, the role of the centrality of each individual in the assimilation process. For example, if the cost of assimilation $c$ is relatively large (as in case (iv) in the proposition), then the less (more) central are the agents, the more (less) likely they will (not) assimilate to the majority culture. This is because the benefits of assimilation (better outcomes in terms of education or employment) outweigh the costs and the more central are the individuals, the more externalities in terms of productivity they obtain from being assimilated. As stated in Section 2, there are very few papers that study the role of networks on assimilation of immigrants. Some papers, such as the ones by Gang and Zimmermann (2000), Furtado and Theodoropoulos (2010), Mouw et al. (2014) and Maani et al. (2015) have examined the impact of immigrant networks on outcomes and find positive effects. An interesting paper is that of Furtado and Theodoropoulos (2010) who show that having access to native networks increases the probability of immigrant employment. This is what is found in Proposition 3 where some minorities are linked to some majorities workers and this helps them become assimilated and improve their productivity and thus their situation in the labor market.

\section{Examples}

\subsection{Star-shaped network}

To illustrate the previous results, consider the star-shaped network $g$ depicted in the following figure: 


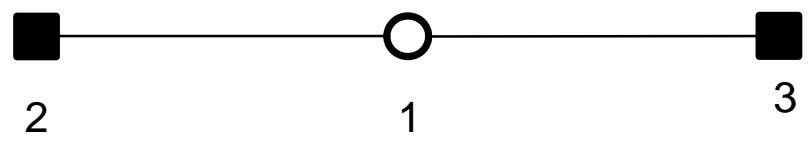

Figure 1: A star network with 3 individuals from different communities

In this figure, circle (white) nodes correspond to the majority group (type $W$ ) while square (black) nodes correspond to the minority group (type $B$ ). There are three agents (i.e. $q+n=3$ ) and agent 1 holds a central position whereas agents 2 and 3 are peripherals. We see that individual 1 belongs to the majority group (type $W$ ) while individuals 2 and 3 belong to the minority group (type $B$ ), i.e. $Q=\{1\}$ and $N=\{2,3\}$. Since there is only one type for the minority workers, i.e. $\omega=1$, there will be only two possible equilibria, which are given by the following proposition:

Proposition 4 (Assimilation choices in a star-shaped network) Consider the star-shaped network depicted in Figure 1 where individual 1 belongs to the majority group and individuals 2 and 3 are minority workers. Assume $\theta<1 / \sqrt{2}$.

(i) If

$$
c<\frac{t(1-\theta)(1+\theta)^{2}[2 \alpha-(1-\theta) t]}{2\left(1-2 \theta^{2}\right)^{2}}
$$

there exists a unique Assimilation equilibrium where all agents are assimilated, i.e. $\mathcal{A}=$ $\{1,2,3\}$ and $\mathcal{O}=\varnothing$.

(ii) If

$$
c>\frac{t(1-\theta)(1+\theta)^{2}[2 \alpha-(1-\theta) t]}{2\left(1-2 \theta^{2}\right)^{2}}
$$

there exists a unique Oppositional equilibrium where all minority workers are oppositional, i.e. $\mathcal{A}=\{1\}$ and $\mathcal{O}=\{2,3\}$.

This proposition shows the role of $c$ and $t$ in the assimilation choices. For fixed values of $\alpha, t$ and $\theta$, when we increase $c$, we switch from an Assimilation equilibrium to an Oppositional equilibrium. As stated above, this is what was found by Adida et al. (2014) who study the assimilation of Muslims in France. In their paper, they measure the cost of assimilation $c$ by the taste-based discrimination of Muslims by the majority group. Similar results are obtained when $t$ decreases. 
In our model, $t$ is the productivity cost of being non-assimilated because, for example, of poorer language skills that affect the productivity of education or on-the-job skill.

Interestingly, for fixed values of $\alpha, t$ and $c$, when we decrease $\theta$, the degree of social interactions, we obtain the same types of result because an increase in $\theta$ means that social interactions are more valuable in terms of outcomes and thus tend to induce people to assimilate more. Let us give some parameter values for which each condition is satisfied given that $\theta<1 / \sqrt{2}=0.707$. For example, for Proposition 4, if we set $\alpha=6, t=1$ and $\theta=0.2$, then: $(i)$ if $c<7.62$, there exists a unique Assimilation equilibrium where $\mathcal{A}=\{1,2,3\}$ and $\mathcal{O}=\varnothing$; (ii) if $c>7.62$, there exists a unique Oppositional equilibrium where $\mathcal{A}=\{1\}$ and $\mathcal{O}=\{2,3\}$.

\subsection{Complete networks}

Let us now consider a complete network where, as in the previous example, we set $q+n=3$. The complete network is displayed in the Figure 2.

\section{Minority group Majority group Minority group}

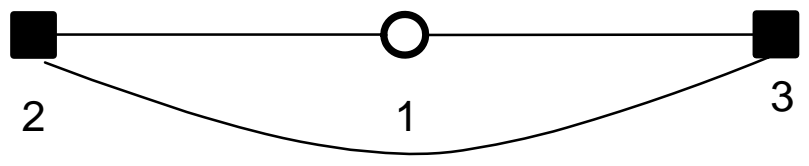

Figure 2: A complete network with three individuals from different communities

Since there is necessary only one type of minority worker, there will always only be the two following equilibria: an Assimilation equilibrium and an Oppositional equilibrium. Assume that agent 1 belongs to the majority group and individuals 2 and 3 to the minority group.

Proposition 5 (Assimilation choices in a complete network) Consider the complete network with 3 agents where individual 1 belongs to the majority group and 2 and 3 are minority workers. Assume that $\theta<1 / 2$.

(i) If

$$
c<\frac{t(1-\theta)^{2}(2 \alpha+4 \alpha t-t)}{2\left(1-\theta-2 \theta^{2}\right)^{2}}
$$

there exists a unique Assimilation equilibrium where all minority agents assimilate, i.e. $\mathcal{A}=$ $\{1,2,3\}$ and $\mathcal{O}=\varnothing$. 
(ii) If

$$
c>\frac{t(1-\theta)^{2}(2 \alpha+4 \alpha t-t)}{2\left(1-\theta-2 \theta^{2}\right)^{2}}
$$

there exists a unique Oppositional equilibrium where all minority agents are oppositional, i.e. $\mathcal{A}=\{1\}$ and $\mathcal{O}=\{2,3\}$.

This proposition completely characterizes the equilibrium configuration for a complete network. We can give parameter values for which each condition is satisfied given that $\theta<1 / 2=0.5$. For example, if take exactly the same parameters as for the star network, i.e. $\alpha=6, t=1$ and $\theta=0.2$, then: $(i)$ if $c<21.61$, there exists a unique Assimilation equilibrium where $\mathcal{A}=\{1,2,3\}$ and $\mathcal{O}=\varnothing ;$; ii $)$ if $c>21.61$, there exists a unique Oppositional equilibrium where $\mathcal{A}=\{1\}$ and $\mathcal{O}=\{2,3\}$.

When we compare the star network and the complete network with 3 agents, where individual 1 belongs to the majority group and 2 and 3 are minority workers, we see that there is much more assimilation among minority workers in the complete network than in the star-shaped network. Indeed, if we again consider the parameters $\alpha=6, t=1$ and $\theta=0.2$, then when $7.62<c<21.61$, all minority workers are assimilated in the complete network while they all oppositional in the star network. This is because there are much more interactions in the complete than in the star network because, in the former, everybody interact directly with everybody while, in the latter, agents 1 and 2 interact directly with the star (agent 1) but only indirectly with each other. This is in fact a general result, which is straightforward to prove, which says that denser networks will have more assimilated individuals than less dense networks. To summarize,

Proposition 6 (Aggregate interactions) Assume $\theta \rho(\mathbf{G})<1$. There is more assimilation in denser networks than in less dense networks.

This result is due to the fact that, because of (local) complementarities in effort in the utility function, aggregate interactions as well as the entire vector of individual interactions increase with the density of the network. ${ }^{6}$ As a result, minority individuals find it more beneficial to assimilate in denser networks. As White et al. (1976) remind us, social networks, by themselves, do not produce a uniformly simple effect, nor are they simply the conduit of contextual influence. Rather, they can facilitate or inhibit assimilation by structuring interactions between initiates and members and by tying both into the larger social structure. At each stage, then, networks constrain or facilitate

\footnotetext{
${ }^{6}$ We define network density as follows. Consider an alternative social network $g^{\prime}, g^{\prime} \neq g$ such that for all $i, j$, $g_{i j}^{\prime}=1$ if $g_{i j}=1$. It is conventional to refer to $g$ and $g^{\prime}$ as nested networks, and to denote their relationship as $g \subset g^{\prime}$. As discussed in Ballester et al. (2006), the network $g^{\prime}$ has a denser structure of network links: some agents who are not directly connected in $g$ are directly connected in $g^{\prime}$.
} 
contact with members, condition members' reactions to initiates, and influence initiates' attitudes, values and beliefs. Proposition 6 formalizes this intuition in terms of network density.

\section{$5 \quad$ Welfare analysis and subsidy policies}

Let us first look at the second stage of the game where all agents decide their optimal effort level. Let us therefore study welfare policies for a given assimilation equilibrium.

\subsection{First-best analysis when assimilation is given}

\subsubsection{First-best analysis}

We would like to see if the equilibrium outcomes are efficient in terms of productive interactions. For that, the planner chooses $x_{1}, \ldots, x_{n}$ to maximize total welfare, that is:

$$
\begin{aligned}
\max _{x_{1}, \ldots, x_{n}} \mathcal{W} & =\max _{x_{1}, \ldots, x_{n}} \sum_{i=1}^{i=q+n} U_{i}\left(x_{i}, \mathbf{x}_{-\mathbf{i}}, g\right) \\
& =\max _{x_{1}, \ldots, x_{n}}\left\{\sum_{i=1}^{i=q+n}\left[\alpha_{i} x_{i}-\frac{1}{2} x_{i}^{2}\right]+\theta \sum_{i=1}^{i=q+n} \sum_{j=1}^{q+n} g_{i j} x_{i} x_{j}\right\}
\end{aligned}
$$

First-order condition gives for each $i=1, \ldots, q+n:^{7}$

$$
\alpha_{i}-x_{i}+\theta \sum_{j} g_{i j} x_{j}+\theta \sum_{j} g_{j i} x_{j}=0
$$

which implies that $\left(\right.$ since $\left.g_{i j}=g_{j i}\right):^{8}$

$$
x_{i}^{O}=\alpha_{i}+2 \theta \sum_{j} g_{i j} x_{j}
$$

Using (2), we easily see that:

$$
x_{i}^{O}=v_{i}^{*}+\theta \sum_{j} g_{i j} x_{j}
$$

where $x_{i}^{*}$ is the Nash equilibrium efforts given in (2). This means that individuals are exerting too little effort at the Nash equilibrium as compared to the social optimum outcome. Equilibrium interaction effort is too low because each agent ignores the positive impact of her effort on the efforts of others, that is, each agent ignores the positive externality arising from complementarity in effort choices. As a result, the market equilibrium is not efficient and the planner would like to subsidize the (productivity) effort of each agent.

\footnotetext{
${ }^{7}$ It is easily checked that there is a unique maximum for each $x_{i}$.

${ }^{8}$ The superscript $O$ refers to the "social optimum" outcome while a star refers to the "Nash equilibrium" outcome.
} 


\subsubsection{Subsidizing social interactions}

Letting $S_{i}^{O}$ denote the optimal subsidy to per effort, comparison of (9) and (10) implies:

$$
S_{i}^{O}=\theta \sum_{j} g_{i j} x_{j}
$$

or in matrix form

$$
\mathbf{S}^{O}=\theta \mathbf{G} \mathbf{x}
$$

If we add one stage before the visit game is played, the planner will announce the optimal subsidy $S_{i}^{O}$ to each agent $i$ such that:

$$
U_{i}=\left(\alpha_{i}+S_{i}^{O}\right) x_{i}-\frac{1}{2} x_{i}^{2}+\theta \sum_{j} g_{i j} x_{i} x_{j}=\alpha_{i} x_{i}-\frac{1}{2} x_{i}^{2}+2 \theta \sum_{j} g_{i j} x_{i} x_{j}
$$

By doing so, the planner will restore the first best. Observe that the optimal subsidy is such that

$$
\mathbf{x}^{O}=(\mathbf{I}-\theta \mathbf{G})^{-1}\left(\alpha_{i}+S_{i}^{O}\right) \mathbf{1}=(\mathbf{I}-2 \theta \mathbf{G})^{-1} \boldsymbol{\alpha}
$$

where $\boldsymbol{\alpha}=\left(\alpha_{1}, \ldots, \alpha_{n}\right)^{\mathrm{T}}$, which means that

$$
x_{i}^{O}=\sum_{j=1}^{q+n} \sum_{k=0}^{+\infty} \theta^{k} g_{i j}^{[k]}\left(\alpha_{j}+S_{j}^{O}\right)=\sum_{j=1}^{q+n} \sum_{k=0}^{+\infty} 2^{k} \theta^{k} g_{i j}^{[k]} \alpha_{j}
$$

and thus

$$
U_{i}^{O}=\frac{1}{2}\left[b_{\alpha+S^{O}}(\theta, g)\right]^{2}=\frac{1}{2}\left[b_{\alpha}(2 \theta, g)\right]^{2}
$$

In particular, the optimal subsidy is given by:

$$
S_{i}^{O}=\theta \sum_{j} g_{i j} x_{j}^{O}=\theta \sum_{j=1}^{q+n} \sum_{l=1}^{q+n} \sum_{k=0}^{+\infty} 2^{k} \theta^{k} g_{i j} g_{j l}^{[k]} \alpha_{l}
$$

What is interesting here is that the planner will give a larger subsidy to more central agents (independently of their ethnicity) in the network. Since we assumed that the individuals from the majority group are more central than the individuals from the minority group, then the planner will subsidize more the activities (in terms of education or work) of the majority group.

Proposition 7 (Optimal level of social interactions) The Nash equilibrium outcome in terms of productive efforts is not efficient since there are too few of them. If the planner proposes a subsidy $S_{i}^{O}=\theta \sum_{j} g_{i j} x_{j}$ to each individual $i$, then the first-best outcome can be restored. In that case, it is optimal for the planner to give higher subsidies to more central agents in the network. 


\subsection{Endogenous choice of assimilation}

\subsubsection{Effort subsidies}

Assume that the planner cannot control assimilation choices but only effort decisions. In that case, in the second stage, she will choose a higher level of effort given by

$$
x_{i}^{O}=\sum_{j=1}^{q+n} \sum_{k=0}^{+\infty} 2^{k} \theta^{k} g_{i j}^{[k]} \alpha_{j}
$$

and then let the minority agents choose whether they want to assimilate or not. In other words, the timing is as follows: First, minority agents choose whether to assimilate or not and then the government chooses the optimal effort for all agents. The second stage is solved as above and the optimal effort is given by (13). We can characterize the equilibrium assimilation and it is clear that more minority agents will assimilate compared to the case when there are no subsidies.

Furthermore, if we investigate a constrained efficient allocation in which the planner can subsidize interactions (i.e., provide a subsidy of $S_{i}^{O}$ per effort by agent $i$ ) but cannot directly control assimilation choices, then more agents will assimilate compared to the case without subsidies. Indeed, since all agents devote more effort to interacting with others under the subsidy program (11), the incentives for assimilation must be stronger under that allocation than in the Subgame-Perfect Nash equilibrium.

Proposition 8 (Equilibrium versus optimal assimilation choices) If the planner proposes a per effort subsidy $S_{i}^{O}$ to each individual $i$, then, compared to the Subgame-Perfect Nash equilibrium described in Proposition 3, more minority agents will assimilate.

\subsubsection{Assimilation subsidies}

Let us now consider the case where the planner subsidizes assimilation but not effort. Since there are more interactions when agents assimilate and since interactions increase utility, then the planner could subsidize the assimilation cost c. For example, the government can make it easier for immigrants to assimilate by helping them moving to more mixed areas ${ }^{9}$ or by having language courses for new immigrants (for example, the Swedish For Immigrants course in Sweden for new immigrants). The law on veiling, by preventing young Muslim women to wear the veil in public areas, could also be seen as a way to help young ethnic minorities to assimilate to the majority culture. ${ }^{10}$

\footnotetext{
${ }^{9}$ For example, the Moving to Opportunity (MTO) programs do that by giving housing vouchers to poor family, usually blacks or Hispanics, to help them move to richer areas. See Ludwig et al. (2001) and Kling et al. (2005).

${ }^{10}$ This is a controversial law and its effects on assimilation are not clear. For example, Carvalho (2013) models the veiling among Muslim women as a form of cultural resistance, which inhibits the transmission of secular values. His
} 
In this policy, the government gives a per-cost subsidy $\sigma$ so that the cost of assimilating would be $(1-\sigma) c$ instead of $c$. The timing is now as follows. In the first stage, the planner announces the subsidy to ethnic minorities who decide to assimilate. In the second stage, agents decide whether they will assimilate or not and, in the last stage, their decide their effort level. As for the subsidy effort, this will clearly generate more assimilation but the mechanism is different since, in the latter, the effect is direct while, in the former, it is indirect. In that case, equilibrium efforts will still be determined by (4) while assimilation decisions will be characterized by Proposition 3 where $c$ has to be replaced by $(1-\sigma) c$.

In this model, it is clear that, if the planner wants to reach the first best in terms of assimilation, she will subsidize $c$ so that all agents will be assimilated. This maximizes aggregate interactions and thus total welfare. For example, in the case of the star network described in Figure 1, where individual 1 is from the majority group and individuals 2 and 3 are from the minority group, we have shown that if $\alpha=6, t=1$ and $\theta=0.2$, then: $(i)$ if $c<7.62$, there exists a unique Assimilation equilibrium; (ii) if $c>7.62$, there exists a unique Oppositional equilibrium. As a result, if, for all agents, $(1-\sigma) c \leq 7.62$, which is equivalent to $\sigma \geq 1-(7.62 / c)$, then the first best is reached and all minority workers assimilate. For example, if $c=20$, then planner needs to subsidize 61.9 percent of the cost of assimilation of all agents. Interestingly, this result depends on the network structure. For the complete network with 3 agents, we have seen that, with exactly the same parameters, $\alpha=6, t=1$ and $\theta=0.2$, then: $(i)$ if $c<21.61$, there exists a unique Assimilation equilibrium; (ii) if $c>21.61$, there exists a unique Oppositional equilibrium. In that case, we need to subsidize $\sigma \geq 1-(21.61) / c$ percent of $c$ for all agents to reach the first best. Thus, for the complete network, if $c=20$, the planner does not need to subsidy any worker to reach the first best in efforts since $20<21.61$. Using this reasoning and looking at Proposition 3 , the optimal subsidy for any network with $n$ minority individuals is given by:

$$
\sigma^{O}>1-\frac{\Phi^{\mathcal{A}}\left(m_{q+n, q+n}\right)}{2 c}
$$

where, from (6), we have:

$$
\Phi^{\mathcal{A}}\left(m_{q+n, q+n}\right) \equiv t(2 \alpha-t)\left(m_{q+n, q+n}\right)^{2}+2 t\left(\alpha \sum_{j=1}^{q+n-1} m_{q+n, q+j}\right) m_{q+n, q+n}
$$

Observe that equation (14) gives the subsidy for the minority individual $q+n$ who has the lowest centrality in the network. Indeed, if the planner gives a $c$-subsidy of $1-\left[\Phi^{\mathcal{A}}\left(m_{q+n, q+n}\right) / 2 c\right]$ to all agents, the first best will be reached since all individuals will be induced to assimilate. This

theory predicts that veiling is highest when individuals from highly religious communities interact in highly secular environments. 
is clearly a sufficient condition. The planner could also discriminate between agents and gives a different subsidy to each agent so that the higher is the centrality of an agent in a network, the lower is the subsidy. In that case, the subsidy to be given to each agent $i$ will be equal to:

$$
\sigma_{i}^{O}>1-\frac{\Phi^{\mathcal{A}}\left(m_{q+i, q+i}\right)}{2 c}
$$

for all $i=1, \ldots, n$, where $\Phi^{\mathcal{A}}\left(m_{q+i, q+i}\right)$ is defined by (6). Of course, this policy is much more complicated to implement (and more costly) because it requires that the planner knows the position of all agents in the network. On the contrary, the previous policy (14) only requires to know the minority worker who has the less central position in the network.

Observe also that if $\Phi^{\mathcal{A}}\left(m_{q+n, q+n}\right)>2 c$, meaning that $1-\frac{\Phi^{\mathcal{A}}\left(m_{q+n, q+n}\right)}{2 c}<0$, the condition (14) is always satisfied. This is because, in this case, we do not need to subsidize any worker to obtain an Assimilation equilibrium because $\Phi^{\mathcal{A}}\left(m_{q+n, q+n}\right)>2 c$ is precisely the condition for which an Assimilation equilibrium exists and is unique (see Proposition 3(i)). Assuming that, when a worker is indifferent between assimilating and not assimilating, she always chooses to assimilate, then the subsidy (16) can be written as:

$$
\sigma_{i}^{O}=\max \left\{0,1-\frac{\Phi^{\mathcal{A}}\left(m_{q+i, q+i}\right)}{2 c}\right\}
$$

\subsubsection{Effort versus assimilation subsidy}

Let us now study both the effort and assimilation subsidies. We have seen that if the planner only subsidizes effort, then the optimal subsidy is given by (12), that is

$$
S_{i}^{O}=\theta \sum_{j=1}^{q+n} \sum_{l=1}^{q+n} \sum_{k=0}^{+\infty} 2^{k} \theta^{k} g_{i j} g_{j l}^{[k]} \alpha_{l}
$$

This optimal subsidy clearly depends on agents' assimilation behaviors. As a result, the first best when both assimilations and efforts are taken into account should be when the effort subsidy is $S_{i}^{O}$ and all agents assimilate. The timing is now as follows. First, the planner announces the assimilation and effort subsidies. Second, agents choose to assimilate or not. Third, agents choose efforts.

Denote $\mathbf{M}^{O}=(\mathbf{I}-2 \theta \mathbf{G})^{-1}$ so that the element of the $i$ th and $j$ th of $\mathbf{M}^{O}$ is $m_{i j}^{O}$. Then, using the same reasoning as above, the assimilation subsidy and the effort subsidy that guarantee that the first best is achieved is determined in the following proposition:

Proposition 9 (First best with effort and assimilation subsidies) Assume $2 \theta \rho(\mathbf{G})<1$ and consider any network of $q+n$ agents. If the assimilation subsidy for minority individuals and the 
effort subsidy for all individuals are such that

$$
\left\{\begin{array}{c}
\sigma_{i}^{O}=\max \left\{0,1-\frac{2 \Phi^{\mathcal{C}}\left(m_{i i}^{O}\right)}{c}\right\}, \text { for } i \in N \\
S_{i}^{O}=\theta \alpha \sum_{j=1}^{q+n} \sum_{l=1}^{q+n} \sum_{k=0}^{+\infty} 2^{k} \theta^{k} g_{i j} g_{j l}^{[k]}, \text { for } i \in Q+N
\end{array}\right.
$$

then all agents choose to assimilate and provide optimal interaction efforts and therefore the first best is achieved.

This proposition implies that, to reach the first best, it is optimal for the planner to give higher effort subsidies but lower assimilation subsidies to more central agents in the social network.

If we consider the star network of Figure 1, it is readily verified that, if $\theta<1 /(2 \sqrt{2})=0.35$, then, if all agents assimilate (i.e. $\alpha_{1}=\alpha_{2}=\alpha_{3}=\alpha$ ), we have:

$$
\mathbf{M}^{O} \boldsymbol{\alpha}=(\mathbf{I}-2 \theta \mathbf{G})^{-1} \boldsymbol{\alpha}=\frac{\alpha}{\left(1-8 \theta^{2}\right)}\left(\begin{array}{c}
1+4 \theta \\
1+2 \theta \\
1+2 \theta
\end{array}\right)
$$

so that

$$
\left(\begin{array}{c}
x_{1}^{O} \\
x_{2}^{O} \\
x_{3}^{O}
\end{array}\right)=\frac{\alpha}{\left(1-8 \theta^{2}\right)}\left(\begin{array}{c}
1+4 \theta \\
1+2 \theta \\
1+2 \theta
\end{array}\right)
$$

Since the optimal effort subsidy for each agent $i$ is given by:

$$
S_{i}^{O}=\theta \sum_{j} g_{i j} x_{j}^{O}
$$

or in matrix form

$$
\mathbf{S}^{O}=\theta \mathbf{G} \mathbf{x}^{O}
$$

We have

$$
\begin{gathered}
S_{1}^{O}=\theta\left(x_{2}^{O}+x_{3}^{O}\right)=2 \alpha \theta\left(\frac{1+2 \theta}{1-8 \theta^{2}}\right) \\
S_{2}^{O}=S_{3}^{O}=\theta x_{1}^{O}=\alpha \theta\left(\frac{1+4 \theta}{1-8 \theta^{2}}\right)
\end{gathered}
$$

Not surprisingly, the planner gives more effort subsidy to more central agents since $S_{1}^{O}>S_{2}^{O}$.

Now, let us calculate the assimilation subsidy. The matrix $\mathbf{M}^{O}$ is given by

$$
\mathbf{M}^{O}=(\mathbf{I}-2 \theta \mathbf{G})^{-1}=\frac{1}{\left(1-8 \theta^{2}\right)}\left(\begin{array}{ccc}
1 & 2 \theta & 2 \theta \\
2 \theta & 1-4 \theta^{2} & 4 \theta^{2} \\
2 \theta & 4 \theta^{2} & 1-4 \theta^{2}
\end{array}\right)
$$


As a result,

$$
\begin{aligned}
\Phi^{\mathcal{A}}\left(m_{q+1, q+1}^{O}\right) & =t(2 \alpha-t)\left(m_{q+1, q+1}^{O}\right)^{2}+2 t \alpha\left(m_{q+1, q+2}^{O}+m_{q+1, q+3}^{O}\right) m_{q+1, q+1}^{O} \\
& =\frac{t[2 \alpha(1+4 \theta)-t]}{\left(1-8 \theta^{2}\right)^{2}}
\end{aligned}
$$

Thus, the optimal subsidy given to agent 1 is equal to:

$$
\sigma_{1}^{O}=\max \left\{0,1-\frac{t[2 \alpha(1+4 \theta)-t]}{2 c\left(1-8 \theta^{2}\right)^{2}}\right\}
$$

Similarly, we have:

$$
\begin{aligned}
\Phi^{\mathcal{A}}\left(m_{q+2, q+2}^{O}\right) & =\Phi^{C}\left(m_{q+3, q+3}^{O}\right)=t(2 \alpha-t)\left(m_{q+2, q+2}^{O}\right)^{2}+2 t \alpha\left(m_{q+2, q+1}^{O}+m_{q+2, q+3}^{O}\right) m_{q+2, q+2}^{O} \\
& =\frac{t\left(1-4 \theta^{2}\right)(1+2 \theta)[2 \alpha-t(1-2 \theta)]}{\left(1-8 \theta^{2}\right)^{2}}
\end{aligned}
$$

Thus, the subsidy $\sigma_{2}^{O}=\sigma_{3}^{O}$ to give to agents 2 and 3 is:

$$
\sigma_{2}^{O}=\max \left\{0,1-\frac{t\left(1-4 \theta^{2}\right)(1+2 \theta)[2 \alpha-t(1-2 \theta)]}{2 c\left(1-8 \theta^{2}\right)^{2}}\right\}
$$

As expected, it is easily verified that $\sigma_{1}^{O}<\sigma_{2}^{O}$, i.e. the planner gives less assimilation subsidy to more central agents.

To summarize:

Proposition 10 (Effort versus assimilation subsidies) Assume $2 \theta \rho(\mathbf{G})<1$ and consider any network of $q+n$ agents. To restore the first best, it is optimal for the planner to give higher effort subsidies (to all individuals) but lower assimilation subsidies (to minority individuals) to more central agents in the social network.

Let us now show, with an example, the importance of social network for this last result. Take again $\alpha=6, t=1$ and $\theta=0.2$, then the planner needs to give the following subsidies:

$$
S_{1}^{O}=4.94 \text { and } S_{2}^{O}=S_{3}^{O}=3.18
$$

and

$$
\sigma_{1}^{O}=\max \left\{0,1-\frac{22.28}{c}\right\} \text { and } \sigma_{2}^{O}=\sigma_{3}^{O}=\max \left\{0,1-\frac{14.50}{c}\right\}
$$

to reach the first best. If, for example, $c=20$, then the planner does not need to subsidize agent 1 but needs to subsidy 27.5 percent of the assimilation cost $c$ for agents 2 and 3 in order for them to assimilate. 
Consider now the complete network with the three agents who assimilate. It is easily verified that, if $\theta<0.25$, then

$$
\begin{gathered}
S_{1}^{O}=S_{2}^{O}=S_{3}^{O}=S^{O}=\frac{2 \alpha \theta(1+2 \theta)}{\left(1-2 \theta-8 \theta^{2}\right)} \\
\sigma_{1}^{O}=\sigma_{2}^{O}=\sigma_{3}^{O}=\sigma^{O}=\max \left\{0,1-\frac{t(1-2 \theta)[2 \alpha(1+2 \theta)-t(1-2 \theta)]}{2 c\left(1-2 \theta-8 \theta^{2}\right)^{2}}\right\}
\end{gathered}
$$

If we take the same parameter values, $\alpha=6, t=1$ and $\theta=0.2$, then $S^{O}=12$ and

$$
\sigma^{O}=\max \left\{0,1-\frac{17.36}{c}\right\}
$$

If we compare the two networks, it easily verified that the planner needs to subsidize much more the social effort of all agents in the complete network (there are more network externalities in the complete network compared to the star network) while, for a given $c$ and for given assimilation subsidies, she needs to subsidize less agent 1 but more agents 2 and 3 in the star network. In terms of network design, this means that the planner would not always like to choose the complete network, even though it is the network that generates most interactions among all possible networks. The optimal network will clearly depend on parameters values and will be, in general, difficult to determine.

\section{Integrated versus segregated communities}

So far, we have assumed that the majority group and the minority group always interact with each other, i.e. they belong to the same social network. We know that this not always true in the real world because, for example, minority and majority individuals do not go to the same school, the same college or do not work in the same workplace or do not live in the same area (segregation). Let us consider the case when this is not the case and analyze its consequences on assimilation behavior. To illustrate the result, let us have the following two complete networks where the two communities are totally separated. 


\section{Minority group}

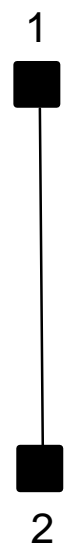

Majority group

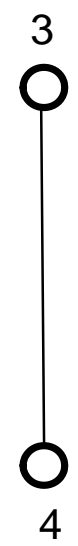

Figure 3: Two separated communities

In Figure 3, circle (white) nodes correspond to the majority group while square (black) nodes correspond to the minority group. Since minority workers 1 and 2 are of the same type (i.e. same centrality), there can only be two equilibria: an Assimilation Equilibrium for which $\mathcal{A}=\{1,2\}$ and $\mathcal{O}=\varnothing$, and an Oppositional Equilibrium for which $\mathcal{A}=\varnothing$ and $\mathcal{O}=\{1,2\}$. Thus, if $\theta<1$, we have:

$$
\mathbf{M}=\frac{1}{\left(1-\theta^{2}\right)}\left(\begin{array}{cc}
1 & \theta \\
\theta & 1
\end{array}\right)
$$

and

$$
\begin{aligned}
\Phi^{\mathcal{A}}\left(m_{11}\right) & =\Phi^{\mathcal{A}}\left(m_{22}\right)=t(2 \alpha-t)\left(m_{11}\right)^{2}+2 t \alpha m_{12} m_{11} \\
& =t(2 \alpha-t)\left(m_{22}\right)^{2}+2 t \alpha m_{21} m_{22}=\frac{t[2 \alpha(1+\theta)-t]}{\left(1-\theta^{2}\right)^{2}}
\end{aligned}
$$

As a result, $(i)$ if $c<\frac{t[2 \alpha(1+\theta)-t]}{2\left(1-\theta^{2}\right)^{2}}$, there is a unique Assimilation Equilibrium while if $c>\frac{t[2 \alpha(1+\theta)-t]}{2\left(1-\theta^{2}\right)^{2}}$, there is a unique Oppositional Equilibrium. The planner would certainly want that both groups interact more since this will increase peer effects and thus total productivity in the economy. Imagine that the planner "creates" a bridge between the two communities so that the two networks are now as follows: 


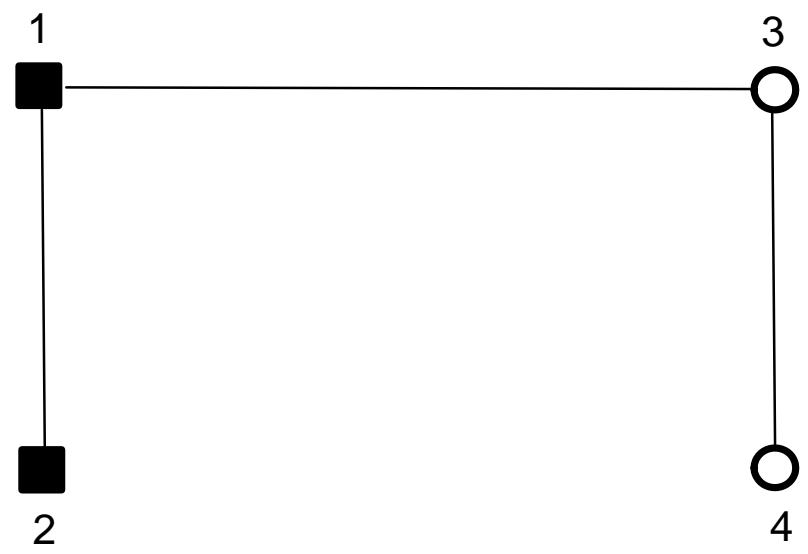

Figure 4: Bridge between the two communities

The planner can create such a bridge by moving minority individuals to more mixed areas (such as Moving to Opportunity programs mentioned above) or by integrating minority individuals to schools or colleges (such as the Affirmative Action or busing policies) or by helping them find a job in "majority" types of jobs. Let us analyze the consequence of this bridge on the assimilation process. Observe first that since the minorities are now of two types (in terms of centralities), there will be three equilibria. If $\theta<\frac{2}{1+\sqrt{5}}=0.618$, we have: ${ }^{11}$

$$
\mathbf{M}_{B}=\frac{1}{\left(1-3 \theta^{2}+\theta^{4}\right)}\left(\begin{array}{cccc}
1-\theta^{2} & \theta\left(1-\theta^{2}\right) & \theta & \theta^{2} \\
\theta\left(1-\theta^{2}\right) & 1-2 \theta^{2} & \theta^{2} & \theta^{3} \\
\theta & \theta^{2} & 1-\theta^{2} & \theta\left(1-\theta^{2}\right) \\
\theta^{2} & \theta^{3} & \theta\left(1-\theta^{2}\right) & 1-2 \theta^{2}
\end{array}\right)
$$

and

$$
\begin{aligned}
\Phi_{B}^{\mathcal{A}}\left(m_{22}\right) & =t(2 \alpha-t)\left(m_{22}\right)^{2}+2 t \alpha\left(m_{21}+m_{23}+m_{24}\right) m_{22} \\
& =\frac{t\left(1-2 \theta^{2}\right)\left[(2 \alpha-t)\left(1-2 \theta^{2}\right)+2 \alpha \theta(1+\theta)\right]}{\left(1-3 \theta^{2}+\theta^{4}\right)^{2}}
\end{aligned}
$$

As a result, if

$$
c<\frac{t\left(1-2 \theta^{2}\right)\left[(2 \alpha-t)\left(1-2 \theta^{2}\right)+2 \alpha \theta(1+\theta)\right]}{2\left(1-3 \theta^{2}+\theta^{4}\right)^{2}}
$$

\footnotetext{
${ }^{11}$ The subscript $B$ indicates that there is a bridge between the two comunities.
} 
there is a unique Assimilation Equilibrium for which $\mathcal{A}=\{1,2\}$ and $\mathcal{O}=\varnothing$. If

$$
\frac{t\left(1-2 \theta^{2}\right)\left[(2 \alpha-t)\left(1-2 \theta^{2}\right)+2 \alpha \theta\left(1-\theta^{2}\right)\right]}{2\left(1-3 \theta^{2}+\theta^{4}\right)^{2}}<c<\frac{t\left(1-\theta^{2}\right)\left[\left(1-\theta^{2}\right)(2 \alpha-t-2 t \theta)+2 \alpha \theta\left(2+\theta-\theta^{2}\right)\right]}{2\left(1-3 \theta^{2}+\theta^{4}\right)^{2}}
$$

there exists a unique Assimilation-Oppositional equilibrium for which $\mathcal{A}=\{1\}$ and $\mathcal{O}=\{2\}$. Finally, if

$$
c>\frac{t\left(1-\theta^{2}\right)\left[\left(1-\theta^{2}\right)(2 \alpha-t-2 t \theta)+2 \alpha \theta\left(2+\theta-\theta^{2}\right)\right]}{2\left(1-3 \theta^{2}+\theta^{4}\right)^{2}}
$$

there exists a unique Oppositional equilibrium for which $\mathcal{A}=\varnothing$ and $\mathcal{O}=\{1,2\}$.

It is clear that there is much more assimilation when there is a bridge between the two communities (Figure 4) than when there is not (Figure 3). To see that, take again the same parameter values as above: $\alpha=6, t=1$ and $\theta=0.2$. If there is no bridge, we have that, if $c<7.27$, there is a unique Assimilation equilibrium while if $c>7.27$, there is a unique Oppositional equilibrium. When there is a bridge, we have that: $(i)$ if $c<7.69$, there is a unique Assimilation equilibrium; (ii) if $7.69<c<9.49$, there is a unique Assimilation-Oppositional equilibrium; (iii) if $c>9.49$, there is a unique Oppositional equilibrium. So, for example, if $7.27<c<7.69$, then in the network with separated communities (Figure 3), all minority workers are oppositional while, in the network with the bridge between the communities (Figure 4), individual 1 is assimilated while individual 2 is oppositional. This is a general result that is true for any network. It is due to the fact that, by creating a bridge between the two communities, positive externalities in terms of effort productivity increase. Indeed, if we look at individual 2, we can check that what she receives from individual 1 (i.e. $\left.m_{21}\right)$ increases from $\frac{\theta}{1-\theta^{2}}$ (before the bridge) to $\frac{\theta\left(1-\theta^{2}\right)}{\left(1-3 \theta^{2}+\theta^{4}\right)}$ (after the bridge is created). Similarly, her own centrality $m_{22}$ (i.e. self-loops) increases from $\frac{1}{\left(1-\theta^{2}\right)}$ to $\frac{1-2 \theta^{2}}{\left(1-3 \theta^{2}+\theta^{4}\right)}$. More generally, when creating a bridge between the two communities, the function $\Phi^{\mathcal{A}}\left(m_{i i}\right)$ that gives the incentive to assimilate always increases for both individuals because the centralities of the individuals from the majority group, i.e. $m_{13}$ and $m_{14}$ for individual 1 and $m_{23}$ and $m_{24}$ for individual 2 , are added to this function (compare (18) and (19)). We have therefore the following general result:

Proposition 11 (Bridge and assimilation) Assume $\theta \rho(\mathbf{G})<1$ and consider any network of $q+n$ agents. Creating bridge(s) between the majority and the minority group always (weakly) increases assimilation.

This proposition demonstrates that assimilation is easier when ethnic minorities interact with individuals from the majority group. This is observed empirically. Using data from the European Community Household Panel, De Palo et al. (2006) study the assimilation of immigrants in the host country society by analyzing a question on the number of interactions between the respondent 
and his/her neighbor, as related to the number of interactions with friends or relatives not living with them. Their results suggest that non-EU migrants tend to socialize less with natives, even after controlling for individual characteristics. They also find that migrants' behavior tends to slowly assimilate to that of native migrants (ethnic minorities). They suggest that education has a significant impact on the type of social activities undertaken by the individuals. More-educated people tend to relate somewhat less with close neighbors, but socialize more intensively with the broader community.

More generally, Proposition 11 shows that, by creating bridges between communities all workers are better off in terms of productivities and thus utilities. This means that, for example, if a planner provides a job to a minority worker that is usually reserved to majority workers (thus creating a bridge between the communities), it does not just increase the productivity and utility of this worker but it also increases the productivity of other minority workers from the same community. In other words, helping one minority individual benefits the whole community she belongs to because of the spillovers it generates. Since network structure plays an important role here, it would always be more efficient to create a bridge link between the most central individual from the minority group and the most central individual from the majority group. This is because we have positive complementarities in efforts so that more central agents provide more positive externalities than less central agents. This is, in turn, positively affects the assimilation behavior of minorities because of the effect on their productivity. The potential gains from bridging different parts of a network were important in the early work of Granovetter (1974) and are central to the notion of structural holes developed by Burt (1994). The latter postulates that social capital is created by a network in which people can broker connections between otherwise disconnected segments. In recent years, a number of empirical studies have shown that individuals or organizations who bridge 'structural holes' in networks gain significant payoff advantages. ${ }^{12}$

\section{Network formation and assimilation choices}

So far, we made two important assumptions that allowed us to have general results: $(i)$ the network is fixed and ( $i i)$ the assimilation choice is binary. Because of these two assumptions, in Proposition 3 , we could have very general results in terms of networks since our results were true for any possible network. The main results of Proposition 3 (assimilation increases in the number of central minority

\footnotetext{
${ }^{12}$ See Burt (1994) and Mehra et al. (2003) for influence of structural positions on promotions and performance evaluation, Podolny and Baron (1997) for work on network positions and mobility, and Ahuja (2000) for the influence of a firm's position in inter-organizational networks on its innovativeness and overall performance. See also Burt (2004) who explores the influence of individual position in social networks in shaping the generation of creative ideas.
} 
agents as well as in the density of the network) are due to the assumption that assimilation leads to increases in marginal utility irrespective of the assimilation choices of other agents in the second stage. In this section, we would like to relax these two assumptions.

First, we would to endogeneize the network. It is, however, well-known that non-cooperative games of network formation with nominal lists of intended links are plagued by coordination problems (Myerson, 1991; Jackson and Wolinsky, 1996; Jackson, 2008; Cabrales et al., 2011). This is why it is extremely hard, if not impossible, to provide a full-fledged characterization of all possible stable networks. We are, however, able to provide a full-characterization of equilibria that have a complete-network structure and then study some specific networks that convey information about the different assimilation patterns that may arise in equilibrium.

Second, we will define the assimilation of an ethnic minority individual by the fraction of her friends who are from the majority group and not as a binary variable as before. In other words, the higher is the number of friends an ethnic minority has, the more assimilated is this person. This is a standard way of measure of assimilation in empirical research. Indeed, researchers have used interethnic marriage (Furtado and Theodoropoulos, 2010; Furtado, 2015) or inter-ethnic friendships (Sigelman and Welch, 1993; Berry, 1997; Ryder et al., 2000; Kao and Joyner, 2004; De Marti and Zenou, 2015; Patacchini and Zenou, 2016) as a measure of assimilation of ethnic minorities.

The timing is now as follows. In the first stage, all individuals form links with individuals from both communities. This corresponds to both the assimilation choice and the network formation. In the second stage, all individuals play the education effort game described in Section 3.1. As usual, we solve the model by backward induction.

\subsection{Model}

As above, there are two communities, the majority group (type $W$ ) and the ethnic minority group (type $B$ ). Each agent belongs exclusively to one of the two communities, $B$ or $W$. The type of individual $i$ is denoted by $\tau(i) \in\{B, W\}$. Let $n^{B}=n$ denote the number of $B$ (minority) individuals in the population and let $n^{W}=q$ denote the number of $G$ (majority) individuals in the population. The network $g$ is a set of all individuals $Q+N$, where $Q=\{1, \ldots, q\}, q \geq 2$ is the set of majority individuals while $N=\{1, \ldots, n\}, n \geq 2$ is set of minority individuals. there are $q+n$ individuals in the economy.

We denote the link of two connected individuals, $i$ and $j$, by $i j$. The set of $i$ 's direct contacts is: $\mathcal{N}_{i}(g)=\left\{j \neq i \mid g_{i j}=1\right\}$, which is of size $n_{i}(g)$. The direct contacts of individual $i$ of the same type is $\mathcal{N}_{i}^{\tau(i)}(g)=\left\{j \neq i, \tau(i)=\tau(j) \mid g_{i j}=1\right\}$, and we denote the cardinality of this set by $n_{i}^{\tau(i)}(g)$. 
Preferences As in the previous section, the utility of $i$ is given by (1):

$$
U_{i}\left(x_{i}, \mathbf{x}_{-\mathbf{i}}, g\right)=\alpha x_{i}-\frac{1}{2} x_{i}^{2}+\theta \sum_{j=1}^{q+n} g_{i j} x_{i} x_{j}-\sum_{j \in \mathcal{N}_{i}(g)} c_{i j}(g)
$$

where $\alpha>0$ is the same for all agents. Indeed, for simplicity, we assume that there is no more premium in terms of productivity for assimilated individuals (captured by $t$ in the previous section). We will see below what are the benefits from assimilation. Furthermore, this utility function in the second stage is still given by (1) but we add the total cost of having links (this does not affect the second-stage game). In other words, all individuals pay the costs $c_{i j}>0$ for maintaining their direct relationships. In Proposition 1, we have shown that, if $\theta \rho(\mathbf{G})<1$ and $\boldsymbol{\alpha}=\alpha \mathbf{1}$, then there is a unique Nash interior equilibrium given by:

$$
\mathbf{x}^{*}=\alpha(\mathbf{I}-\theta \mathbf{G})^{-1} \mathbf{1}=\alpha \mathbf{b}(g, \theta)
$$

or

$$
x_{i}^{*}\left(\alpha_{i}, \boldsymbol{\alpha}_{-\mathbf{i}}, g\right)=\alpha b_{i}(g, \theta)
$$

where $b_{i}(g, \theta)$ is now the unweighted Katz-Bonacich centrality (since $\alpha$ is the same for all agents). As a result, the equilibrium utility of each individual $i=B, W$ is now given by (see (5)):

$$
\begin{aligned}
U_{i}^{*}\left(x_{i}^{*}, \mathbf{x}_{-\mathbf{i}}^{*}, g\right) & =\frac{1}{2}\left[x_{i}^{*}\left(\alpha_{i}, \boldsymbol{\alpha}_{-\mathbf{i}}, g\right)\right]^{2}-\sum_{j \in \mathcal{N}_{i}(g)} c_{i j}(g) \\
& =\frac{\alpha^{2}}{2}\left[b_{i}(g, \theta)\right]^{2}-\sum_{j \in \mathcal{N}_{i}(g)} c_{i j}(g)
\end{aligned}
$$

For the exposition, denote $U_{i}^{*}(g) \equiv U_{i}^{*}\left(x_{i}^{*}, \mathbf{x}_{-\mathbf{i}}^{*}, g\right)$. To characterize linking costs, given a network $g$, we define the rate of exposure of individual $i$ to her own community $\tau(i)$ as:

$$
e_{i}^{\tau(i)}(g)=\frac{n_{i}^{\tau(i)}(g)}{n_{i}(g)}
$$

This ratio $e_{i}^{\tau(i)}(g)$ measures the fraction of same-type friends of individual $i$ in network $g$ since $n_{i}^{\tau(i)}(g)$ is the number of $i$ 's same-type friends in network $g$ while $n_{i}(g)$ is the total number of $i$ 's friends in network $g$, independently of their type. We can now introduce the cost structure. Let $c$ and $C$ be strictly positive constants. We assume that: ${ }^{13}$

$$
c_{i j}(g)=\left\{\begin{array}{cl}
c & \text { if } \tau(i)=\tau(j) \\
c_{i j}=c+e_{i}^{\tau(i)} e_{j}^{\tau(j)} C & \text { if } \tau(i) \neq \tau(j)
\end{array}\right.
$$

\footnotetext{
${ }^{13}$ Eguia (2015) and De Marti and Zenou (2015) also present a theory in which the cost of assimilation is endogenous and depends on the choice of others. Their model is, however, very different from ours.
} 
There are thus different costs, depending with whom a connection is made. Since $C>0$ and the rates of exposure are non-negative, the main feature of this cost structure is that it is always more costly to form a friendship relationship with someone from the other community than with someone from the same community. In particular, if an individual $i$ of type $\tau(i)$ forms a friendship relationship with an individual $j$ of type $\tau(j)$, with $\tau(i) \neq \tau(j)$, then the cost is increasing in their respective rates of exposure to their own communities. In other words, it is always easier for ethnic minorities to interact with ethnic minorities and likewise for whites, and that the interethnic relationships strongly depend on how "exposed" individuals are to their own community, i.e. how many same-race friends they have. These difficulties in inter-ethnic relationships can be due to language barriers or more generally to different social norms and cultures, as highlighted in the Introduction and Section 2. This is also what has been found empirically. For example, using data from the German Socio-Economic Panel for the period 1996-2011, Facchini et al. (2015) document that migrants with a German friend are more similar to natives than those without a local companion along several important dimensions, including engagement in social activities, concerns about the economy, interest in politics and broad policy issues like the environment, crime and xenophobia.

As a result, we will say that the higher is the percentage of type- $W$ friends for a type $-B$ individual, the more assimilated is this person. We do not need, as in the previous section, to define who is assimilated and who is not. We could clearly do it by saying that, if a minority individual has at least 50 percent of her friends who are of the other type, then she is considered as assimilated. But this is an arbitrary definition and depends on the size of each population.

In this new formulation, the cost of assimilation is now given by $c_{i j}$, which is the cost of forming a link with someone from both communities. Here the benefits of assimilation is captured in a different way compared to the previous section. If a person from the minority group (type $B$ ) have many links with persons from the majority group (type $W$ ), she will be considered as more assimilated and the benefits will be that her exposure rate increases and, as a result, her cost $c_{i j}$ of forming new links with someone from the majority group will decrease. ${ }^{14}$

Example To illustrate our cost function (22), consider the following network:

\footnotetext{
${ }^{14}$ As stated above, we could introduce the benefits of assimilation in terms of productivity $t$ as in the previous section but then we would need some arbitrary definition of assimilation, i.e. percentage of type- $W$ friends above which someone is assimilated. We could also assume that the benefits from a link with a majority individual are higher than with a minority worker. This would add more notations but will not change any of our results.
} 


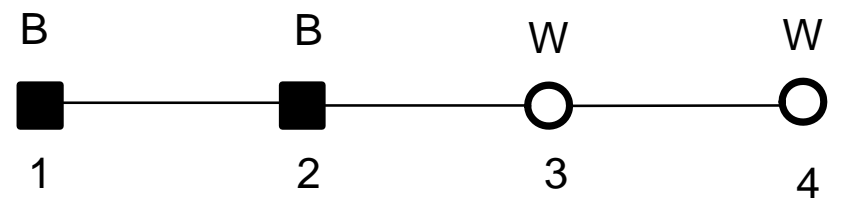

Figure 5: Line network with minority and majority individuals

where circle (white) nodes correspond to the majority group (type $W$ ) while square (black) nodes correspond to the minority group (type $B$ ). Now, assume that only links 12 and 34 exist and that individual 2 (type $B$ ) considers forming a link with individual 3 (type $W$ ). In that case, the cost of connecting individual 2 to individual 3 is:

$$
c_{23}(g)=c+\frac{n_{2}^{\tau(1)}(g)}{n_{2}(g)} \frac{n_{3}^{\tau(3)}(g)}{n_{3}(g)} C=c+C
$$

since $n_{2}^{\tau(2)}(g)=n_{3}^{\tau(3)}(g)=1$ (number of same-type friends of 2 and 3 , respectively) and $n_{2}(g)=$ $n_{3}(g)=1$ (total number of 1's and 3's friends independently of type, before the link 32 is formed), which implies that $e_{1}^{\tau(1)}(g)=e_{3}^{\tau(3)}(g)=1$. It is clear that, in this example, the cost of the link 23 is maximum since both individuals 2 and 3 have not been exposed at all to people from the other community when considering forming the link 23. If for example, we add an extra link with someone of type $W$ for individual 2, then the cost $c_{23}(g)$ decreases and is given by:

$$
c_{23}(g)=c+\frac{1}{2} C
$$

since $e_{2}^{\tau(2)}(g)$, the rate of exposure of individual 2 to her own community, decreases from 1 to $1 / 2$.

\subsection{Network stability}

In games played in a network, individuals' payoffs depend on the network structure. In our case, this dependency is established in expression (20), that encompasses both the benefits and costs attributed to an individual given her position in the network of relationships. Any equilibrium notion introduces some stability requirements. The notion of pairwise-stability, introduced by Jackson and Wolinsky (1996), provides a widely used solution concept in networked environments. Denote by $g+i j$ the network $g$ for which the link $i j \notin g$ has been added and by $g-i j$ the network $g$ for which the link $i j \in g$. has been removed. 
Definition 1 A network $g$ is pairwise stable if and only if:

(i) for all $i j \in g, U_{i}(g) \geq U_{i}(g-i j)$ and $U_{j}(g) \geq U_{j}(g-i j)$

(ii) for all ij $\notin g$, if $U_{i}(g)<U_{i}(g+i j)$ then $U_{j}(g)>U_{j}(g+i j)$.

In words, a network is pairwise-stable if $(i)$ no player gains by cutting an existing link, and $(i i)$ no two players not yet connected both gain by creating a direct link with each other. Pairwisestability thus only checks for one-link deviations. It requires that any mutually beneficial link be formed at equilibrium but does not allow for multi-link severance. In other words, it takes into account the individual incentives to create and sever links and the necessary mutual consent between both sides for a link to be formed.

We will use this equilibrium concept throughout this section. Thus, network $g$ is an equilibrium network whenever it is pairwise stable.

\subsection{Equilibrium}

Let us now characterize the pairwise-stable equilibria for which each community is fully intraconnected (i.e. each individual is linked to all other individuals within the same community) and for which they are totally separated (no links between communities). Denote

$\Gamma\left(\theta, n^{W}, n^{B}\right) \equiv \frac{\alpha^{2}\left(\theta\left[1-\phi\left(n^{W}-1\right)\right]+\theta^{2}\right)\left(2(1+\theta)\left[1-\phi\left(n^{B}-1\right)\right]+\theta\left[1-\phi\left(n^{W}-1\right)+\theta\right]\right)}{2(1+\theta)^{2}\left[1-\phi\left(n^{B}-1\right)\right]^{2}\left[1-\phi\left(n^{W}-1\right)\right]^{2}}$

We have the following result: ${ }^{15}$

Proposition 12 Assume $\theta<1 /(n-1)$ and $n^{B}<n^{W}$. Denote by $m \equiv n+q$ the total number of individuals in the economy.

(i) If

$$
c+\frac{\left(n^{B}-1\right)\left(n^{W}-1\right)^{2}}{(m-1)^{2}(m-2)} C<\frac{\alpha^{2} \theta\left(2+3 \theta+\theta^{2} m\right)[1-\theta(m-2)]}{2(1+\theta)^{4}[1-\theta(m-1)]^{2}}
$$

there exists a unique pairwise-stable network such that the minority and the majority communities are completely integrated, i.e. both communities are completely connected.

(ii) If

$$
\Gamma\left(\theta, n^{W}, n^{B}\right)<c+\frac{\left(n^{B}-1\right)\left(n^{W}-1\right)}{n^{B} n^{W}} C
$$

\footnotetext{
${ }^{15}$ The proof of all the propositions in this section can be found in Appendix 2.
} 
and

$$
c<\frac{\alpha^{2} \theta\left(2+3 \theta+\theta^{2} n^{B}\right)\left[1-\theta\left(n^{B}-2\right)\right]}{2(1+\theta)^{4}\left[1-\theta\left(n^{B}-1\right)\right]^{2}}
$$

there exists a unique pairwise-stable network such that the minority and the majority communities are completely separated, i.e. there is no link between the two communities.

This is an interesting and general result that gives different conditions under which the network displays complete integration, i.e. each community is fully intra-connected and both communities are fully inter-connected, and under which the community are totally separated. As can be seen from the proof of this proposition, these are results that are difficult to establish. This is because, when someone considers either to delete or to add a link, she will change the Katz-Bonacich centralities of all agents in the network and will also change her exposure rate and thus her costs of inter-ethnic links.

Consider $(i)$. We only need to check deviations from deleting links since nobody can form new links. However, we need to check four deviations: a link deleted with someone from the same community and with someone from the other community for both types $W$ and $B$ agents. Consider, for example, the case when a type $-B$ wants to severe a link with a type $-W$ individual. First, one has to calculate the loss in terms of benefits, that is the (negative) change in terms of Katz-Bonacich centrality of this agent, which depends on the Katz-Bonacich centralities of all agents in the network. Second, one also needs to calculate the gains in terms of costs. When a type $-B$ agent deletes a link with a type $-W$ individual, there is a direct gain from this deletion but there is also a loss with all the other type $-W$ agents since the exposure rate of the type $-B$ agent is now higher and thus the costs to maintain links with all type- $W$ agents are higher. This is what is captured by condition (23), which depends on the size of each community, $n^{W}$ and $n^{B}$, and the $\operatorname{costs} c$ and $C$ (which have to be low enough) and on $\theta$, the strength of interactions.

If we now consider $(i i)$, the results are even more complicated to obtain since we need to consider deviations from deleting a link for each agent but also the possibility for both a type- $W$ and a type $-B$ individual to create a bridge link with the other community. Condition (23) is satisfied if both $c$ and $C$ are low enough while conditions (24) and (25) are satisfied for intermediate values of $c$ and high values of $C$. This is because we need to guarantee that both link creation and deletion are not possible.

There is an interesting literature in psychology (see, in particular, Phinney, 1990; Berry, 1997; Ryder et al., 2000) that proposes a broader concept of self-identification in a two-dimensional framework, where identifications with two different cultures are not necessary mutually exclusive. Berry (1997) presents four distinct strategies for how individuals relate to two cultures. Assimilation is a weak identification with the culture of origin and a strong identification with the alternative 
culture. Integration is achieved when an individual combines strong dedication to the culture of origin and large commitment to the other culture. Marginalization is a weak dedication to both cultures. Finally, separation is an exclusive commitment to the culture of origin. Figure 6 summarizes these four different possibilities in a two-dimensional space.

As a result, in Proposition $12(i)$, we have shown that ethnic minorities are integrated since they have not only a strong identification to the majority culture (a large fraction of their friends are of type $W$ ) but also to their own culture (a large fraction of their friends are of type $B$ ). In (ii), we have shown that the ethnic minorities are separated since they have a weak identification to the majority culture but a strong identification to their own culture. In other words, Proposition 12 shows under which condition we can have two opposite behaviors in terms of assimilation choices.

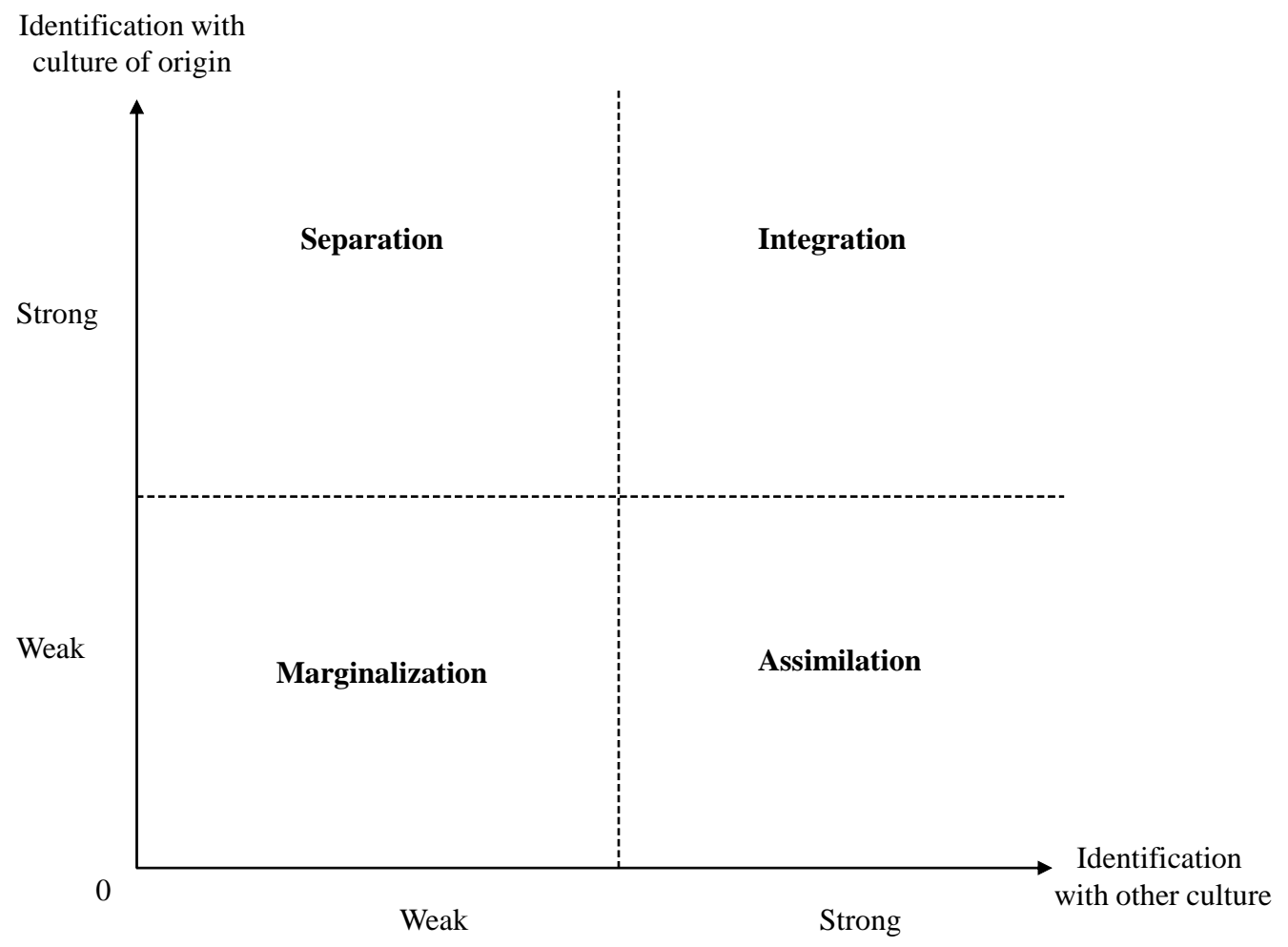

Figure 6. Different identifications for ethnic minorities

An important difference with the model of the previous section (Proposition 3) is that, now, it is not true anymore that assimilation leads to increases in marginal utility irrespective of the assimilation choices of other agents. Here, the choice of friends is endogeneous and the assimilation decision (percentage of friends of type $W$ ) strongly depends on the assimilation choices of other individuals from the majority and the minority group. This is because social distance expresses the force underlying the cost structure. Two agents are closer in the social space, the more each 
of them is exposed to the other community. And, the closer they are in the social space, the easier it is for them to interact. In our model, this social distance is endogenous and depends on the respective choice of peers.

\subsection{Examples}

It is difficult to have more general results because we need to calculate the marginal gain of forming or deleting links and this depends on the difference in Katz-Bonacich centralities. We can, however, give some conditions under which some specific networks are pairwise stables. In order to compare with the previous section, let us take the examples of Section 4.

\subsubsection{Star-shaped network}

Consider the star-shaped network $g$ depicted in Figure 1 where individual 1, who belongs to the majority group, holds a central position, while individuals 2 and 3, who belong to the minority group, are peripherals.

Proposition 13 (Assimilation choices in a star-shaped network) Consider the star-shaped network depicted in Figure 1 where individual 1 belongs to the majority group and individuals 2 and 3 are minority workers. Assume $\theta<1 / 2$. Then this network is an equilibrium (pairwise-stable) network if

$$
\frac{\theta\left(2-\theta-4 \theta^{2}\right)}{\left(1-2 \theta-2 \theta^{2}+4 \theta^{3}\right)^{2}}<\frac{2 c}{\alpha^{2}}<\frac{\theta^{2}\left(2-3 \theta^{2}\right)}{\left(1-\theta-2 \theta^{2}+2 \theta^{3}\right)^{2}}
$$

As in the previous section (Proposition 4), this proposition shows the role of $c, \alpha$ and $\theta$ in the assimilation choices. If $c$ and $\alpha$ have intermediate values, then the star network of Figure 1 is an equilibrium network because individual 1 (type $W$ ) has no incentive to delete a link while individual 2 or 3 (type $B$ ) has no incentive to delete or create a link. In this equilibrium, both minority individuals are assimilated (see Figure 5) since they have a strong identification with the host majority culture since all their friends are from the majority group but a very weak identification with their own culture (no friend from the same community). As in Proposition 4, increasing $\theta$, the intensity of social interactions, will always lead to more assimilation (i.e. more friends for the minority group) because they will be more interactions between all agents. This is not what condition (26) is capturing since if $\theta$ is too large, then individuals 2 and 3 would form a link and the network depicted in Figure 1 will not anymore be pairwise stable. 


\subsubsection{Complete network}

Consider now the complete network $g$ depicted in Figure 2 where individual 1, who belongs to the majority group, holds a central position, while individuals 2 and 3, who belong to the minority group, are peripherals.

Proposition 14 (Assimilation choices in a complete network) Consider the complete network depicted in Figure 2 where individual 1 belongs to the majority group and individuals 2 and 3 are minority workers. Assume $\theta<1 / 2$. Then this network is an equilibrium (pairwise-stable) network if

$$
\frac{2 c}{\alpha^{2}}<\frac{\theta\left(2-\theta-4 \theta^{2}\right)}{\left(1-2 \theta-2 \theta^{2}+4 \theta^{3}\right)^{2}}
$$

Condition (27) implies that the lower is $c$ or the higher is $\alpha$, the more likely that the complete network described in Figure 2 will emerge. Moreover, when $\theta<1 / 2$, it is easily verified that the right-hand side of condition (27) is increasing in $\theta$ so that the higher is $\theta$, the more valuable are social interactions in the network and the more likely the complete network will be formed.

Observe that, for these two specific networks of Figures 1 and 2, the inter-ethnic cost is always equal to the intra-ethnic cost, i.e. $c$, because individual 1 from the majority group has no friend from the majority group and her exposure rate was always zero. This considerably simplifies the proof of Propositions 13 and 14. Of course, in richer networks, this will not be the case (see e.g. Proposition 12). Consider, for example, the network of Figure 5. If agent 2 (type $B$ ) wants to severe her link with agent 3 (type $W$ ), the gain is not anymore $c$, as in Propositions 13 and 14, but $c+C / 4$ since, by deleting link 23, the exposure rate of individual 2 to her own community increases from $1 / 2$ to 1 and the total cost of her links decreases from $2 c+C / 4$ (costs of the links 12 and 13) to $c$ (cost of the link 12).

This proposition is interesting because it provides policy implications similar to the ones proposed in Section 6. Indeed, if the government could decrease $c$, and, more generally $C$ (see Proposition 12) the intra- and inter-ethnic cost of interactions, for example, by having language courses to immigrants, or increase $\alpha$, the workers' productivity, or $\theta$, social interactions, then assimilation will increase.

\section{Concluding remarks}

In this paper, we have proposed a simple two stage-model where ethnic minorities choose whether to assimilate or not to the majority culture in the first stage while, in the second stage, both ethnic minorities and majority workers decide how much effort to put in some activity. The network is 
taken as given. We show that the more central agents in the network tend to assimilate more than the less central agents. We also show that there is more assimilation in denser networks and the Nash equilibrium is not efficient because of positive externalities created by effort choices. We then propose different policies that can restore the first best, looking at both effort and assimilation subsidies. We also show that bridging networks between the majority and the majority group is always beneficial for assimilation behaviors.

We then extend our model to include network formation and, instead of having a binary choice of assimilation, we have a more "continuous" definition of assimilation, which is endogenously determined by the percentage of friends from the majority group each minority worker has. Using the concept of pairwise stability for equilibrium networks, we provide conditions for which the ethnic minorities either assimilate to or separated themselves from the majority group.

As a result, there is a trade off between the two models. In the first one, which has a simple view of the assimilation process and for which the network is exogenously given, we can derive several general results. In the second one, where the position in the network of each individual as well as the number of friends of both types are endogenous, we can only provide some partial results, especially for specific networks such as the complete ones. However, both of them lead to the policy conclusion that integrating the two communities (by either moving minorities from areas with many minority families to areas with many majority families, as, for example, in the MTO programs in the United States, and/or by reducing the cost of assimilation and the cost of interaction between communities) leads to more assimilation. In particular, both show that there will be more assimilation if there are more inter-ethnic links in the network. Therefore, our results suggest that ethnic enclaves may not be good for assimilation while providing host country language proficiency could be a more effective policy. ${ }^{16}$ They also suggest that targeting the most central individuals among the minority group (such as community leaders) could be an efficient policy if they are themselves assimilated because this will generate positive externalities in terms of assimilation behaviors and outcomes for minority individuals in the network.

\section{References}

[1] Adida, C.L., Laitin, D.D. and M.-A. Valfort (2014), "Muslims in France: Identifying a discriminatory equilibrium," Journal of Population Economics 27, 1039-1086.

\footnotetext{
${ }^{16}$ Interestingly, this is also the conlusion of Angelini et al. (2015) who empirically investigate the association between assimilation with a host culture and immigrants' subjective well-being in Germany.
} 
[2] Ahuja, G. (2000), "Collaboration networks, structural holes, and innovation: A logitudinal study," Administrative Science Quarterly 45, 425-455.

[3] Ainsworth-Darnell, J.W. and D.B. Downey (1998), "Assessing the oppositional culture explanation for racial/ethnic differences in school performance," American Sociological Review 63, 536-553.

[4] Akerlof, G. (1997), "Social distance and social decisions," Econometrica 65, 1005-1027.

[5] Akerlof, G.A. and R.E. Kranton (2010), Identity Economics: How Our Identities Shape Our Work, Wages, and Well-Being, Princeton: Princeton University Press.

[6] Alba, R.D., Kasinitz, P. and M.C. Waters (2011), "The kids are (mostly) alright: Secondgeneration assimilation," Social Forces 89, 763-774.

[7] Alba, R.D. and V. Nee (2003), Remaking the American Mainstream: Assimilation and Contemporary Immigration, Cambridge, MA: Harvard University Press.

[8] Angelini, V., Casi, L. and L. Corazzini (2015), "Life satisfaction of immigrants: Does cultural assimilation matter?" Journal of Population Economics 28, 817-844.

[9] Austen-Smith, D. and R.D. Fryer, Jr (2005), "An economic analysis of 'acting white'," Quarterly Journal of Economics 120, 551-583.

[10] Ballester, C., Calvó-Armengol, A. and Y. Zenou (2006), "Who's who in networks. Wanted: The key player," Econometrica 74, 1403-1417.

[11] Bartlett, M.S. (1951), "An inverse matrix adjustment arising in discriminant analysis," Annals of Mathematical Statististics 22, 107-111.

[12] Battu, H., McDonald, M. and Y. Zenou (2007), "Oppositional identities and the labor market," Journal Population Economics 20, 643-667.

[13] Battu, H. and Y. Zenou (2010), "Oppositional identities and employment for ethnic minorities. Evidence for England," Economic Journal 120, 52-71.

[14] Beenstock, M., Chiswick, B.R. and A. Paltiel (2010), "Testing the immigrant assimilation hypothesis with longitudinal data," Review of Economics of the Household 8, 7-27.

[15] Berry, J.W. (1997), "Immigration, acculturation and adaptation," Applied Psychology: An International Review 46, 5-34. 
[16] Bisin, A., Patacchini, E., Verdier, T. and Y. Zenou (2011a), "Formation and persistence of oppositional identities," European Economic Review 55, 1046-1071.

[17] Bisin, A., Patacchini, E., Verdier, T. and Y. Zenou (2011b), "Ethnic identity and labor-market outcomes of immigrants in Europe," Economic Policy 26, 57-92.

[18] Bisin, A. and T. Verdier (2000), "Beyond the melting pot: Cultural transmission, marriage, and the evolution of ethnic and religious traits," Quarterly Journal of Economics 115, 955-988.

[19] Bisin, A. and T. Verdier (2001), "The economics of cultural transmission and the dynamics of preferences," Journal of Economic Theory 97, 298-319.

[20] Bisin, A. and T. Verdier (2011), "The economics of cultural transmission and socialization," In J. Benhabib, A. Bisin, and M.O. Jackson (Eds.), Handbook of Social Economics, Amsterdam: Elsevier Science, pp. 339-416.

[21] Bonacich, P. (1987), "Power and centrality: A family of measures," American Journal of Sociology 92, 1170-1182.

[22] Borjas G.J. (1985), "Assimilation, changes in cohort quality, and the earnings of immigrants," Journal of Labor Economics 3, 463-489.

[23] Borjas, G.J. (1987), "Self-selection and the earnings of immigrants," American Economic Review $77,531-553$.

[24] Borjas, G.J. (1992), "Ethnic capital and intergenerational mobility," Quarterly Journal of Economics 107, 123-150.

[25] Boyd, R. and P. Richerson (1985), Culture and the Evolutionary Process, Chicago: University of Chicago Press.

[26] Burt, R.S. (1994), Structural Holes, New York: Academic Press.

[27] Burt, R.S (2004), "Structural holes and good ideas," American Journal of Sociology 110, 349-399.

[28] Cabrales, A., Calvó-Armengol, A. and Y. Zenou (2011), "Social interactions and spillovers," Games and Economic Behavior 72, 339-360.

[29] Cavalli-Sforza, L. and M. Feldman (1981), Cultural Transmission and Evolution. A Quantitative Approach, Princeton: Princeton University Press. 
[30] Calvó-Armengol, A., Patacchini, E. and Y. Zenou (2009), "Peer effects and social networks in education," Review of Economic Studies 76, 1239-1267.

[31] Carvalho, J.-P. (2013), "Veiling," Quarterly Journal of Economics 128, 337-370.

[32] Chiswick, B.R. and P.W. Miller (1995), "The endogeneity between language and earnings: International analyses," Journal of Labor Economics 13, 246-288.

[33] Chiswick, B.R. and P.W. Miller (1996), "Ethnic networks and language proficiency among immigrants," Journal of Population Economics 9, 19-35.

[34] Chiswick, B.R. and P.W. Miller (2011), "The 'negative' assimilation of immigrants: A special case," Industrial and Labor Relations Review 64, 502-525.

[35] Constant, A., Gataullina, L. and K.F. Zimmermann (2009), "Ethnosizing immigrants," Journal of Economic Behavior and Organization 69, 274-287.

[36] Constant, A.F. and K.F. Zimmermann (2008), "Measuring ethnic identity and its impact on economic behavior," Journal of the European Economic Association 6, 424-433.

[37] Constant, A.F. and K.F. Zimmermann (2009), "Work and money: Payoffs by ethnic identity and gender," Research in Labor Economics 29, 3-30.

[38] Delpit, L. (1995), Other People's Children: Cultural Conflict in the Classroom. The Free Press, New York.

[39] De Marti, J. and Y. Zenou (2015), "Segregation in friendship networks," Unpublished manuscript, Stockholm University.

[40] De Palo, D., Faini, R. and A. Venturini (2006), "The social assimilation of immigrants," IZA Discussion Paper No. 2439.

[41] Dequiedt, V. and Y. Zenou (2014), "Local and consistent centrality measures in networks," Unpublished manuscript, Stockholm University.

[42] Dustmann C. (1994), "Speaking fluency, writing fluency and earnings of migrants," Journal of Population Economics 7, 133-156.

[43] Dustmann C. (1996), "The social assimilation of immigrants," Journal of Population Economics $9,37-54$. 
[44] Dustmann, C. and F. Fabbri (2003), "Language proficiency and labour market performance of immigrants in the UK," Economic Journal 113, 695-717.

[45] Edin, P.A., Fredriksson, P. and O. Aslund (2003), "Ethnic enclaves and the economic success of immigrants - Evidence from a natural experiment," Quarterly Journal of Economics 118, 329-357.

[46] Eguia, J.X. (2015), "Discrimination and assimilation," Unpublished manuscript, Michigan State University.

[47] Facchini, G., Patacchini, E. and M.F. Steinhardt (2015), "Migration, friendship ties and cultural assimilation," Scandinavian Journal of Economics 117, 619-649.

[48] Fang, H. and G.C. Loury (2005), "Dysfunctional identities can be rational," American Economic Review 95, 104-111.

[49] Fordham, S. and J. Ogbu (1986), "Blacks students' school successes: Coping with the burden of 'acting white'," The Urban Review 18, 176-206.

[50] Fryer, R.G. Jr. and P. Torelli (2010), "An empirical analysis of 'acting white'," Journal of Public Economics 94, 380-396.

[51] Furtado, D. and N. Theodoropoulos (2010), "Why does intermarriage increase immigrant employment? The role of networks," B.E. Journal of Economic Analysis $\mathcal{E}$ Policy 10(3), Article 101.

[52] Furtado, D. and S. Trejo (2013), "Interethnic marriages and their economic effects," In: A.F. Constant and K.F. Zimmermann (Eds.), International Handbook on the Economics of Migration, Cheltenham: Edward Elgar, pp. 276-92.

[53] Furtado, D. (2015), "Ethnic intermarriage," In: J.D. Wright (Ed.), International Encyclopedia of Social and Behavioral Sciences, 2nd Edition, Oxford: Elsevier Science, pp. 118-122.

[54] Gang, I.N. and K.F. Zimmermann (2000), "Is child like parent? Educational attainment and ethnic origin," Journal of Human Resources 35, 550-569.

[55] Giulietti, C., Wahba, J. and Y. Zenou (2014), "Strong versus weak ties in migration", CEPR Discussion Paper No. 10248.

[56] Gorinas, C. (2014), "Ethnic identity, majority norms, and the native-immigrant employment gap," Journal of Population Economics 27, 225-250. 
[57] Granovetter, M.S. (1974), Getting a Job: A Study of Contacts and Careers, Cambridge, MA: Harvard University Press.

[58] Haller, W., Portes, A. and S. Lynch (2011), "On the dangers of rosy lenses," Social Forces 89, 775-782.

[59] Helsley, R.W. and Y. Zenou (2014), "Social networks and interactions in cities," Journal of Economic Theory 150, 426-466.

[60] Ioannides, Y.M. (2012), From Neighborhoods to Nations: The Economics of Social Interactions, Princeton: Princeton University Press.

[61] Jackson, M.O. (2008), Social and Economic Networks, Princeton: Princeton University Press.

[62] Jackson, M.O., Rogers, B. and Y. Zenou (2015), "The economic consequences of social network structure", CEPR Discussion Paper 10406.

[63] Jackson, M.O and A. Wolinsky (1996), "A strategic model of social and economic networks," Journal of Economic Theory 71, 44-74.

[64] Jackson, M.O. and Y. Zenou (2015), "Games on networks", In: P. Young and S. Zamir (Eds.), Handbook of Game Theory, Vol. 4, Amsterdam: Elsevier Publisher, pp. 91-157.

[65] Kahanec, M. and K.F. Zimmermann (2011), Ethnic Diversity in European Labor Market. Challenges and Solutions, Cheltenham: Edward Elgar.

[66] Kao, G. and K. Joyner (2004), "Do race and ethnicity matter among friends? Activities among interracial, interethnic, and intraethnic adolescent friends," Sociological Quarterly 45, 557-574.

[67] Katz, L. (1953), "A new status index derived from sociometric analysis," Psychometrika 18, 39-43.

[68] Kling, J.R., Ludwig, J. and L.F. Katz (2005), "Neighborhood effects on crime for female and male youth: Evidence from a randomized housing voucher experiment," Quarterly Journal of Economics 120, 87-130.

[69] Laitin, D.D. (1995), "Marginality: A microperspective," Rationality and Society 7, 31-57.

[70] Lazear E. (1999), "Culture and language", Journal of Political Economy 107, S95-S126.

[71] Ludwig, J., Duncan, G.J. and P. Hirschfield (2001), "Urban poverty and juvenile crime: Evidence from a randomized housing-mobility experiment," Quarterly Journal of Economics 116, 655-679. 
[72] Mehra, A., Kilduff, M. and D. Bass (2003), "The social networks of high and low self-monitors: Implications for workplace performance," Administrative Science Quarterly 46, 121-146.

[73] Mouw, T., Chavez, S., Edelblute, H. and A. Verdery (2014), "Binational social networks and assimilation: A test of the importance of transnationalism," Social Problems 61, 329-359.

[74] Maani, S.A., Wang, X. and A. Rogers (2015), "Network effects, ethnic capital and immigrants' earnings assimilation: Evidence from a spatial, Hausman-Taylor estimation," IZA Discussion Paper No. 9308.

[75] McManus, W., Gould, W. and F. Welch (1983), "Earnings of Hispanic men: The role of English language proficiency," Journal of Labor Economics 1, 101-103.

[76] Myerson, R.B. (1991), Game Theory: Analysis of Conflict, Harvard: Harvard University Press.

[77] Nguyen Le, A. (2010), "Assimilating through social networks? The importance of networks in assimilation trajectories," Unpublished manuscript, University of British Columbia, Department of Sociology.

[78] Ogbu, J.U. (1997), "Beyond language: Ebonics, proper English and identity in a black American speech community," Unpublished manuscript, University of California at Berkeley, Department of Anthropology.

[79] Panebianco, F. (2014), "Socialization networks and the transmission of interethnic attitudes," Journal of Economic Theory 150, 583-610.

[80] Patacchini, E. and Y. Zenou (2016), "Racial identity and education in social networks," Social Networks 44, 85-94.

[81] Phinney, J.S. (1990), "Ethnic identity in adolescents and adults: Review of research," Psychological Bulletin 108, 499-514.

[82] Podolny, J and J. Baron (1997), "Resources and relationships, social networks and mobility in the work place," American Sociological Review 62, 673-693.

[83] Portes, A. and R.G. Rumbaut (2001), Legacies, Berkeley: University of California Press.

[84] Portes, A. and M. Zhou (1993), "The new second generation: Segmented assimilation and its variants," Annals of the American Academy of Political and Social Science 530, 74-96. 
[85] Ryder A.G., Alden, L.E. and D.L. Paulhus (2000), "Is acculturation unidimensional or bidimensional? A head-to-head comparison in the prediction of personality, self-identity, and adjustment," Journal of Personality and Social Psychology 79, 49-65.

[86] Selod, H. and Y. Zenou (2006), "Does city structure affect the labour market outcomes of black workers?" Economic Journal 116, 1057-1087.

[87] Sigelman, L. and S. Welch (1993), "The contact hypothesis revisited: Black-White interaction and positive racial attitudes," Social Forces 71, 781-795.

[88] Verdier, T. and Y. Zenou (2015), "Cultural leaders and the dynamics of assimilation," Unpublished manuscript, Stockholm University.

[89] White, H., Boorman, S. and R. Breiger (1976), "Social structure from multiple networks I. Blockmodels of roles and positions," American Journal of Sociology 81, 730-80.

[90] Wilson, W.J. (1987), The truly Disadvantaged: The Inner City, the Underclass, and Public Policy, Chicago: University of Chicago Press.

[91] Zhou, M. and C.L. Bankston (1998), Growing up American: How Vietnamese Children Adapt to Life in the United States, New York: Russell Sage Foundation.

[92] Zimmermann, K.F. (2007), "The economics of migrant ethnicity," Journal of Population Economics 20, 487-494.

[93] Zimmermann, L., Zimmermann, K.F. and A. Constant (2007), "Ethnic self-identification of first-generation immigrants," International Migration Review 41, 769-781. 


\section{Appendix 1: The Katz-Bonacich network centrality measure}

The Katz-Bonacich centrality measure (due to Katz, 1953, and Bonacich, 1987) has proven to be extremely useful in game theoretical applications (Ballester et al., 2006; Dequiedt and Zenou, 2014). $\mathbf{G}$ is the $n \times n$ adjacency matrix that keeps track of the direct connections in the network. Let $\mathbf{G}^{k}$ be the $k$ th power of $\mathbf{G}$, with elements $g_{i j}^{[k]}$, where $k$ is an integer. The matrix $\mathbf{G}^{k}$ keeps track of the indirect connections in the network: $g_{i j}^{[k]} \geq 0$ gives the number of walks or paths of length $k \geq 1$ from $i$ to $j$ in the network $g$. In particular, $\mathbf{G}^{0}=\mathbf{I}$. Consider the matrix $\mathbf{M}=\sum_{k=0}^{+\infty} \theta^{k} \mathbf{G}^{k}$. The elements of this matrix, $m_{i j}$, count the number of walks of all lengths from $i$ to $j$ in the network $g$, where walks of length $k$ are weighted by $\theta^{k}$. The unweighted Katz-Bonacich centrality of agent $i$, denoted, $b_{i}(g, \theta)$ is equal to the sum of the elements of the $i$ th row of $\mathbf{M}$ :

$$
b_{i}(g, \theta)=\sum_{j=1}^{n} m_{i j}=\sum_{j=1}^{n} \sum_{k=0}^{+\infty} \theta^{k} g_{i j}^{[k]} .
$$

Note that the Katz-Bonacich centrality is well-defined if $\theta$ is low enough. In matrix form, the vector of Katz-Bonacich centralities can be written as:

$$
\mathbf{b}(g, \theta)=\mathbf{M} \mathbf{1}=\left[\mathbf{I}_{n}-\theta \mathbf{G}\right]^{-1} \mathbf{1}_{n},
$$

where $\mathbf{I}_{n}$ is the $n \times n$ identity matrix and $\mathbf{1}_{n}$ is the $n$-vector of ones. We can also define the weighted Katz-Bonacich centrality of agent $i$ as:

$$
b_{\alpha_{i}}(g, \theta)=\sum_{j=1}^{n} \sum_{k=0}^{+\infty} \theta^{k} g_{i j}^{[k]} \alpha_{j}
$$

where the weight attached to the walks from $i$ to $j$ is $\alpha_{j}$. For any $n$-dimensional vector $\boldsymbol{\alpha}$, i.e. $\boldsymbol{\alpha}=\left(\alpha_{1}, \ldots, \alpha_{n}\right)^{\mathrm{T}}$, where $\mathrm{T}$ stands for the transpose vector, the matrix equivalent of (30) is given by:

$$
\mathbf{b}_{\boldsymbol{\alpha}}(g, \theta)=\mathbf{M} \boldsymbol{\alpha}=\left[\mathbf{I}_{n}-\theta \mathbf{G}\right]^{-1} \boldsymbol{\alpha}
$$

Denote by $g_{n}^{C}$, with corresponding adjacency matrix $\mathbf{G}_{n}^{C}$, the complete network with $n$ agents. We have the following result:

Lemma 1 Assume $\theta<1 /(n-1)$. For the complete network with $n$ agents, the weighted KatzBonacich centrality is equal to:

$$
\mathbf{b}_{\boldsymbol{\alpha}}\left(g_{n}^{C}, \theta\right)=\frac{1}{1+\theta} \boldsymbol{\alpha}+\frac{\theta \sum_{i=1}^{i=n} \alpha_{i}}{(1+\theta)[1-\theta(n-1)]} \mathbf{1}_{n}
$$


while the unweighted Katz-Bonacich centrality is given by:

$$
\mathbf{b}\left(g_{n}^{C}, \theta\right)=\frac{\alpha}{1-\theta(n-1)} \mathbf{1}_{n}
$$

Proof. First, the largest eigenvalue of a complete graph is $n-1$ and therefore the condition for the Katz-Bonacich to be well-defined is $\theta<1 /(n-1)$. Second, observe that the adjacency matrix of the complete network with $n$ agents, $\mathbf{G}_{n}^{C}$ is equal to:

$$
\mathbf{G}_{n}^{C}=\mathbf{1}_{n} \mathbf{1}_{n}^{\mathrm{T}}-\mathbf{I}_{n}
$$

where $\mathbf{1}_{n}^{\mathrm{T}}$ is the transpose of the vector $\mathbf{1}_{n}$. This is because $\mathbf{G}_{n}^{C}$ has a zero diagonal and a 1 everywhere else. As a result, the weighted vector of Katz-Bonacich centralities is given by:

$$
\begin{aligned}
\mathbf{b}_{\boldsymbol{\alpha}}\left(g_{n}^{C}, \theta\right) & =\left[\mathbf{I}_{n}-\theta \mathbf{G}_{n}^{C}\right]^{-1} \boldsymbol{\alpha} \\
& =\left[\mathbf{I}_{n}-\theta\left(\mathbf{1}_{n} \mathbf{1}_{n}^{\mathrm{T}}-\mathbf{I}_{n}\right)\right]^{-1} \boldsymbol{\alpha} \\
& =\left[(1+\theta) \mathbf{I}_{n}+\left(-\theta \mathbf{1}_{n} \mathbf{1}_{n}^{\mathrm{T}}\right)\right]^{-1} \boldsymbol{\alpha}
\end{aligned}
$$

Now, using the Sherman and Morrison's formula (see Bartlett, 1951, p. 107), we have:

$$
\begin{aligned}
& {\left[(1+\theta) \mathbf{I}_{n}+\left(-\theta \mathbf{1}_{n} \mathbf{1}_{n}^{\mathrm{T}}\right)\right]^{-1} } \\
= & {\left[(1+\theta) \mathbf{I}_{n}\right]^{-1}-\frac{\left[(1+\theta) \mathbf{I}_{n}\right]^{-1}\left(-\theta \mathbf{1}_{n} \mathbf{1}_{n}^{\mathrm{T}}\right)\left[(1+\theta) \mathbf{I}_{n}\right]^{-1}}{1+\left(-\theta \mathbf{1}_{n}^{\mathrm{T}}\right)\left[(1+\theta) \mathbf{I}_{n}\right]^{-1} \mathbf{1}_{n}} } \\
= & \frac{1}{1+\theta} \mathbf{I}_{n}+\frac{\frac{\theta}{(1+\theta)^{2}} \mathbf{1}_{n} \mathbf{1}_{n}^{\mathrm{T}}}{1-\frac{\theta}{1+\theta} n} \\
= & \frac{1}{1+\theta} \mathbf{I}_{n}+\frac{\theta}{(1+\theta)^{2}} \frac{1+\theta}{1-\theta(n-1)} \mathbf{1}_{n} \mathbf{1}_{n}^{\mathrm{T}} \\
= & \frac{1}{1+\theta}\left(\mathbf{I}_{n}+\frac{\theta}{1-\theta(n-1)} \mathbf{1}_{n} \mathbf{1}_{n}^{\mathrm{T}}\right)
\end{aligned}
$$

Hence,

$$
\begin{aligned}
\mathbf{b}_{\boldsymbol{\alpha}}\left(g_{n}^{C}, \theta\right) & =\left[\mathbf{I}_{n}-\theta \mathbf{G}_{n}^{C}\right]^{-1} \boldsymbol{\alpha} \\
& =\left[(1+\theta) \mathbf{I}_{n}+\left(-\theta \mathbf{1}_{n} \mathbf{1}_{n}^{\mathrm{T}}\right)\right]^{-1} \boldsymbol{\alpha} \\
& =\frac{1}{1+\theta}\left(\mathbf{I}_{n}+\frac{\theta}{1-\theta(n-1)} \mathbf{1}_{n} \mathbf{1}_{n}^{\mathrm{T}}\right) \boldsymbol{\alpha} \\
& =\frac{1}{1+\theta} \boldsymbol{\alpha}+\frac{\theta \sum_{i=1}^{i=n} \alpha_{i}}{(1+\theta)[1-\theta(n-1)]} \mathbf{1}_{n}
\end{aligned}
$$


If agents are ex ante identical so that $\boldsymbol{\alpha}=\alpha \mathbf{1}_{n}$, then we obtain the unweighted vector of KatzBonacich centralities:

$$
\begin{aligned}
\mathbf{b}\left(g_{n}^{C}, \theta\right) & =\frac{\alpha}{1+\theta} \mathbf{1}_{n}+\frac{\theta n \alpha}{(1+\theta)[1-\theta(n-1)]} \mathbf{1}_{n} \\
& =\frac{\alpha}{1-\theta(n-1)} \mathbf{1}_{n}
\end{aligned}
$$

This completes the proof. 


\section{Appendix 2: Proof of Propositions}

\section{Proof of Proposition 12:}

We denote by $m=n+q=n^{B}+n^{W}$ the total number of individuals in the economy.

(i) We want to show that the network for which each agent has $m-1$ links (the two communities are totally integrated) is pairwise stable. We don't need to check for link-formation deviation since nobody can create a link because the total network is complete. However, each person can delete a link. When an individual $i$ deletes a link, the loss is equal to: $B_{i}^{*}\left(g_{m}^{C}-i j\right)-B_{i}^{*}\left(g_{m}^{C}\right)$, where $g_{m}^{C}$ denotes the complete network with $m$ agents, $g_{m}^{C}-i j$, the network $g_{m}^{C}$ where the link $i j$ has been removed and $B_{i}^{*}(g)$, the positive part (benefits) of the equilibrium utility function (20), i.e. $B_{i}^{*}(g) \equiv \frac{\alpha^{2}}{2}\left[b_{i}(g, \theta)\right]^{2}$. We have:

$$
\begin{aligned}
B_{i}^{*}\left(g_{m}^{C}-i j\right)-B_{i}^{*}\left(g_{m}^{C}\right) & =\frac{\alpha^{2}}{2}\left[b_{i}\left(g_{m}^{C}-i j, \theta\right)\right]^{2}-\frac{\alpha^{2}}{2}\left[b_{i}\left(g_{m}^{C}, \theta\right)\right]^{2} \\
& =\frac{\alpha^{2}}{2}\left[b_{i}\left(g_{m}^{C}-i j, \theta\right)-b_{i}\left(g_{m}^{C}, \theta\right)\right]\left[b_{i}\left(g_{m}^{C}-i j, \theta\right)+b_{i}\left(g_{m}^{C}, \theta\right)\right]
\end{aligned}
$$

Let us first calculate $b_{i}\left(g_{m}^{C}-i j, \theta\right)-b_{i}\left(g_{m}^{C}, \theta\right)$, which the (negative) change in the Katz-Bonacich centrality for individual $i$ when she removes the link $i j$ from the complete graph with $n$ agents. Denote by $\mathbf{G}_{m}^{C-i j}$ the adjacency matrix of the network $g_{m}^{C}-i j$. We have

$$
\mathbf{G}_{m}^{C-i j}=\mathbf{1}_{m} \mathbf{1}_{m}^{\mathrm{T}}-\mathbf{e}_{i} \mathbf{e}_{j}^{\mathrm{T}}-\mathbf{I}_{m}
$$

where $\mathbf{e}_{i}$ is the $m$-vector who has $i$ th component equal to 1 and the rest 0 . Remember from Appendix 1 that the weighted Katz-Bonacich centrality is equal to:

$$
\mathbf{b}_{\boldsymbol{\alpha}}(g, \theta)=\mathbf{M} \boldsymbol{\alpha}=\left[\mathbf{I}_{m}-\theta \mathbf{G}\right]^{-1} \boldsymbol{\alpha}
$$

As a result, we need to calculate $\left[\mathbf{I}_{m}-\theta \mathbf{G}_{m}^{C-i j}\right]^{-1}$. We have:

$$
\begin{aligned}
{\left[\mathbf{I}_{m}-\theta \mathbf{G}_{m}^{C-i j}\right]^{-1} } & =\left[\mathbf{I}_{m}-\theta\left(\mathbf{1}_{m} \mathbf{1}_{m}^{\mathrm{T}}-\mathbf{e}_{i} \mathbf{e}_{j}^{\mathrm{T}}-\mathbf{I}_{m}\right)\right]^{-1} \\
& =\left[\left((1+\theta) \mathbf{I}_{m}+\left(-\theta \mathbf{1}_{m} \mathbf{1}_{m}^{\mathrm{T}}\right)\right)+\theta \mathbf{e}_{i} \mathbf{e}_{j}^{\mathrm{T}}\right]^{-1}
\end{aligned}
$$


Using the Sherman and Morrison's formula (see Bartlett, 1951, p. 107), we obtain:

$$
\begin{aligned}
& {\left[\left((1+\theta) \mathbf{I}_{m}+\left(-\theta \mathbf{1}_{m} \mathbf{1}_{m}^{\mathrm{T}}\right)\right)+\theta \mathbf{e}_{i} \mathbf{e}_{j}^{\mathrm{T}}\right]^{-1} } \\
= & {\left[(1+\theta) \mathbf{I}_{m}+\left(-\theta \mathbf{1}_{m} \mathbf{1}_{m}^{\mathrm{T}}\right)\right]^{-1}-\frac{\left[(1+\theta) \mathbf{I}_{m}+\left(-\theta \mathbf{1}_{m} \mathbf{1}_{m}^{\mathrm{T}}\right)\right]^{-1}\left(\theta \mathbf{e}_{i} \mathbf{e}_{j}^{\mathrm{T}}\right)\left[(1+\theta) \mathbf{I}_{m}+\left(-\theta \mathbf{1}_{m} \mathbf{1}_{m}^{\mathrm{T}}\right)\right]^{-1}}{1+\left(\theta \mathbf{1}_{m}^{\mathrm{T}}\right)\left[(1+\theta) \mathbf{I}_{m}+\left(-\theta \mathbf{1}_{m} \mathbf{1}_{m}^{\mathrm{T}}\right)\right]^{-1} \mathbf{1}_{m}} } \\
= & \frac{1}{1+\theta}\left[\mathbf{I}_{m}+\frac{\theta}{1-\theta(m-1)} \mathbf{1}_{m} \mathbf{1}_{m}^{\mathrm{T}}\right]-\frac{\frac{\theta}{(1+\theta)^{2}}\left[\mathbf{I}_{m}+\frac{\theta}{1-\theta(m-1)} \mathbf{1}_{m} \mathbf{1}_{m}^{\mathrm{T}}\right] \mathbf{e}_{i} \mathbf{e}_{j}^{\mathrm{T}}\left[\mathbf{I}_{m}+\frac{\theta}{1-\theta(m-1)} \mathbf{1}_{m} \mathbf{1}_{m}^{\mathrm{T}}\right]}{1+\frac{\theta}{1+\theta} \mathbf{1}_{m}^{\mathrm{T}}\left[\mathbf{I}_{m}+\frac{\theta}{1-\theta(m-1)} \mathbf{1}_{m} \mathbf{1}_{m}^{\mathrm{T}}\right] \mathbf{1}_{m}} \\
= & \frac{1}{1+\theta}\left[\mathbf{I}_{m}+\frac{\theta}{1-\theta(m-1)} \mathbf{1}_{m} \mathbf{1}_{m}^{\mathrm{T}}\right]-\frac{\frac{\theta}{(1+\theta)^{2}}\left[\mathbf{e}_{i} \mathbf{e}_{j}^{\mathrm{T}}+\frac{\theta}{1-\theta(m-1)}\left(\mathbf{1}_{m} \mathbf{e}_{j}^{\mathrm{T}}+\mathbf{e}_{i} \mathbf{1}_{m}^{\mathrm{T}}\right)+\frac{\theta^{2}}{(1-\theta(m-1))^{2}} \mathbf{1}_{m} \mathbf{1}_{m}^{\mathrm{T}}\right]}{1+\frac{\theta}{1+\theta}\left[m+\frac{\theta}{1-\theta(m-1)} m^{2}\right]}
\end{aligned}
$$

Observe that the denominator of the second term is equal to:

$$
\begin{aligned}
1+\frac{\theta}{1+\theta}\left[m+\frac{\theta}{1-\theta(m-1)} m^{2}\right] & =1+\frac{m \theta}{1+\theta}\left[1+\frac{m \theta}{1-\theta(m-1)}\right] \\
& =\frac{1+\theta}{1-\theta(m-1)}
\end{aligned}
$$

Therefore,

$$
\begin{aligned}
{\left[\mathbf{I}_{m}-\theta \mathbf{G}_{m}^{C-i j}\right]^{-1}=} & \frac{1}{1+\theta}\left[\mathbf{I}_{m}+\frac{\theta}{1-\theta(m-1)} \mathbf{1}_{m} \mathbf{1}_{m}^{\mathrm{T}}\right] \\
& -\frac{\frac{\theta}{(1+\theta)^{2}}\left[\mathbf{e}_{i} \mathbf{e}_{j}^{\mathrm{T}}+\frac{\theta}{1-\theta(m-1)}\left(\mathbf{1}_{m} \mathbf{e}_{j}^{\mathrm{T}}+\mathbf{e}_{i} \mathbf{1}_{m}^{\mathrm{T}}\right)+\frac{\theta^{2}}{(1-\theta(m-1))^{2}} \mathbf{1}_{m} \mathbf{1}_{m}^{\mathrm{T}}\right]}{\frac{1+\theta}{1-\theta(m-1)}}
\end{aligned}
$$

From the proof of Lemma 1, we know that:

$$
\left[\mathbf{I}_{m}-\theta \mathbf{G}_{m}^{C}\right]^{-1}=\frac{1}{1+\theta}\left(\mathbf{I}_{m}+\frac{\theta}{1-\theta(m-1)} \mathbf{1}_{m} \mathbf{1}_{m}^{\mathrm{T}}\right)
$$

As a result,

$$
\left[\mathbf{I}_{m}-\theta \mathbf{G}_{m}^{C-i j}\right]^{-1}=\left[\mathbf{I}_{m}-\theta \mathbf{G}_{m}^{C}\right]^{-1}-\frac{\frac{\theta}{(1+\theta)^{2}}\left[\mathbf{e}_{i} \mathbf{e}_{j}^{\mathrm{T}}+\frac{\theta}{1-\theta(m-1)}\left(\mathbf{1}_{m} \mathbf{e}_{j}^{\mathrm{T}}+\mathbf{e}_{i} \mathbf{1}_{m}^{\mathrm{T}}\right)+\frac{\theta^{2}}{(1-\theta(m-1))^{2}} \mathbf{1}_{m} \mathbf{1}_{m}^{\mathrm{T}}\right]}{\frac{1+\theta}{1-\theta(m-1)}}
$$

Thus

$$
\begin{aligned}
& {\left[\mathbf{I}_{m}-\theta \mathbf{G}_{m}^{C-i j}\right]^{-1}-\left[\mathbf{I}_{m}-\theta \mathbf{G}_{m}^{C}\right]^{-1} } \\
= & -\frac{\frac{\theta}{(1+\theta)^{2}}\left[\mathbf{e}_{i} \mathbf{e}_{j}^{\mathrm{T}}+\frac{\theta}{1-\theta(m-1)}\left(\mathbf{1}_{m} \mathbf{e}_{j}^{\mathrm{T}}+\mathbf{e}_{i} \mathbf{1}_{m}^{\mathrm{T}}\right)+\frac{\theta^{2}}{(1-\theta(m-1))^{2}} \mathbf{1}_{m} \mathbf{1}_{m}^{\mathrm{T}}\right]}{\frac{1+\theta}{1-\theta(m-1)}} \\
= & -\frac{\theta[1-\theta(m-1)]}{(1+\theta)^{3}}\left[\mathbf{e}_{i} \mathbf{e}_{j}^{\mathrm{T}}+\frac{\theta}{1-\theta(m-1)}\left(\mathbf{1}_{m} \mathbf{e}_{j}^{\mathrm{T}}+\mathbf{e}_{i} \mathbf{1}_{m}^{\mathrm{T}}\right)+\frac{\theta^{2}}{(1-\theta(m-1))^{2}} \mathbf{1}_{m} \mathbf{1}_{m}^{\mathrm{T}}\right]
\end{aligned}
$$


while

$$
\begin{aligned}
& {\left[\mathbf{I}_{m}-\theta \mathbf{G}_{m}^{C-i j}\right]^{-1}+\left[\mathbf{I}_{m}-\theta \mathbf{G}_{m}^{C}\right]^{-1} } \\
= & \frac{2}{1+\theta}\left(\mathbf{I}_{m}+\frac{\theta}{1-\theta(m-1)} \mathbf{1}_{m} \mathbf{1}_{m}^{\mathrm{T}}\right) \\
& -\frac{\theta[1-\theta(m-1)]}{(1+\theta)^{3}}\left[\mathbf{e}_{i} \mathbf{e}_{j}^{\mathrm{T}}+\frac{\theta}{1-\theta(m-1)}\left(\mathbf{1}_{m} \mathbf{e}_{j}^{\mathrm{T}}+\mathbf{e}_{i} \mathbf{1}_{m}^{\mathrm{T}}\right)+\frac{\theta^{2}}{(1-\theta(m-1))^{2}} \mathbf{1}_{m} \mathbf{1}_{m}^{\mathrm{T}}\right]
\end{aligned}
$$

Consequently, we have:

$$
\begin{aligned}
& \mathbf{b}_{\boldsymbol{\alpha}}\left(g_{m}^{C}-i j, \theta\right)-\mathbf{b}_{\boldsymbol{\alpha}}\left(g_{m}^{C}, \theta\right) \\
& =\left[\mathbf{I}_{m}-\theta \mathbf{G}_{m}^{C-i j}\right]^{-1} \boldsymbol{\alpha}-\left[\mathbf{I}_{m}-\theta \mathbf{G}_{m}^{C}\right]^{-1} \boldsymbol{\alpha} \\
& =\left(\left[\mathbf{I}_{m}-\theta \mathbf{G}_{m}^{C-i j}\right]^{-1}-\left[\mathbf{I}_{m}-\theta \mathbf{G}_{m}^{C}\right]^{-1}\right) \boldsymbol{\alpha} \\
& =-\frac{\theta[1-\theta(m-1)]}{(1+\theta)^{3}}\left[\alpha_{j} \mathbf{e}_{i}+\frac{\theta}{1-\theta(m-1)}\left(\alpha_{j} \mathbf{1}_{m}+\left(\sum_{i=1}^{i=m} \alpha_{i}\right) \mathbf{e}_{i}\right)+\frac{\theta^{2} \sum_{i=1}^{i=m} \alpha_{i}}{(1-\theta(m-1))^{2}} \mathbf{1}_{m}\right] \\
& =-\frac{\theta[1-\theta(m-1)]}{(1+\theta)^{3}}\left[\left(\frac{\alpha_{j} \theta}{1-\theta(m-1)}+\frac{\theta^{2} \sum_{i=1}^{i=m} \alpha_{i}}{[1-\theta(m-1)]^{2}}\right) \mathbf{1}_{m}+\left(\alpha_{j}+\frac{\theta \sum_{i=1}^{i=m} \alpha_{i}}{1-\theta(m-1)}\right) \mathbf{e}_{i}\right]
\end{aligned}
$$

When $\boldsymbol{\alpha}=\alpha \mathbf{1}_{n}$, for $\alpha>0$, the difference in terms of the unweighted Katz-Bonacich centrality is given by:

$$
\begin{aligned}
& \mathbf{b}\left(g_{m}^{C}-i j, \theta\right)-\mathbf{b}\left(g_{m}^{C}, \theta\right) \\
= & \alpha\left[\mathbf{I}_{m}-\theta \mathbf{G}_{m}^{C-i j}\right]^{-1} \mathbf{1}_{m}-\alpha\left[\mathbf{I}_{m}-\theta \mathbf{G}_{m}^{C}\right]^{-1} \mathbf{1}_{m} \\
= & -\frac{\theta[1-\theta(m-1)]}{(1+\theta)^{3}}\left[\left(\frac{\alpha \theta}{1-\theta(m-1)}+\frac{\theta^{2} m \alpha}{[1-\theta(m-1)]^{2}}\right) \mathbf{1}_{n}+\left(\alpha+\frac{\theta m \alpha}{1-\theta(m-1)}\right) \mathbf{e}_{i}\right] \\
= & -\frac{\theta[1-\theta(m-1)]}{(1+\theta)^{3}}\left[\frac{\alpha \theta(1+\theta)}{[1-\theta(m-1)]^{2}} \mathbf{1}_{n}+\frac{\alpha(1+\theta)}{1-\theta(m-1)} \mathbf{e}_{i}\right] \\
= & -\frac{\alpha \theta}{(1+\theta)^{2}}\left[\frac{\theta}{1-\theta(m-1)} \mathbf{1}_{m}+\mathbf{e}_{i}\right]
\end{aligned}
$$

As a result, when $\boldsymbol{\alpha}=\alpha \mathbf{1}_{n}$, for individual $i$, the difference in terms of the unweighted Katz-Bonacich centrality is equal to:

$$
\begin{aligned}
b_{i}\left(g_{m}^{C}-i j, \theta\right)-b_{i}\left(g_{m}^{C}, \theta\right) & =-\frac{\alpha \theta}{(1+\theta)^{2}}\left[\frac{\theta}{1-\theta(m-1)}+1\right] \\
& =-\frac{\alpha \theta}{(1+\theta)^{2}}\left[\frac{1-\theta(m-2)}{1-\theta(m-1)}\right]
\end{aligned}
$$


Finally, the difference of the unweighted Katz-Bonacich centralities for which $\alpha=1$ for all agents is given by:

$$
b_{i}\left(g_{m}^{C}-i j, \theta\right)-b_{i}\left(g_{m}^{C}, \theta\right)=-\frac{\theta}{(1+\theta)^{2}}\left[\frac{1-\theta(m-2)}{1-\theta(m-1)}\right]
$$

Let us now calculate $b_{i}\left(g_{m}^{C}-i j, \theta\right)+b_{i}\left(g_{m}^{C}, \theta\right)$. Using (33), we have:

$$
\begin{aligned}
{\left[\mathbf{I}_{m}-\theta \mathbf{G}_{m}^{C}\right]^{-1}+\left[\mathbf{I}_{m}-\theta \mathbf{G}_{m}^{C-i j}\right]^{-1}=} & \frac{2}{1+\theta}\left[\mathbf{I}_{m}+\frac{\theta}{1-\theta(m-1)} \mathbf{1}_{m} \mathbf{1}_{m}^{\mathrm{T}}\right] \\
& -\frac{\frac{\theta}{(1+\theta)^{2}}\left[\mathbf{e}_{i} \mathbf{e}_{j}^{\mathrm{T}}+\frac{\theta}{1-\theta(m-1)}\left(\mathbf{1}_{m} \mathbf{e}_{j}^{\mathrm{T}}+\mathbf{e}_{i} \mathbf{1}_{m}^{\mathrm{T}}\right)+\frac{\theta^{2}}{(1-\theta(m-1))^{2}} \mathbf{1}_{m} \mathbf{1}_{m}^{\mathrm{T}}\right]}{\frac{1+\theta}{1-\theta(m-1)}}
\end{aligned}
$$

Therefore,

$$
\begin{aligned}
& \mathbf{b}_{\boldsymbol{\alpha}}\left(g_{m}^{C}, \theta\right)+\mathbf{b}_{\boldsymbol{\alpha}}\left(g_{m}^{C}-i j, \theta\right) \\
& =\left[\mathbf{I}_{m}-\theta \mathbf{G}_{m}^{C}\right]^{-1} \boldsymbol{\alpha}+\left[\mathbf{I}_{m}-\theta \mathbf{G}_{m}^{C-i j}\right]^{-1} \boldsymbol{\alpha} \\
& =\left(\frac{2}{1+\theta}\left(\mathbf{I}_{m}+\frac{\theta}{1-\theta(m-1)} \mathbf{1}_{m} \mathbf{1}_{m}^{\mathrm{T}}\right)\right) \boldsymbol{\alpha} \\
& -\left(\frac{\frac{\theta}{(1+\theta)^{2}}\left[\mathbf{e}_{i} \mathbf{e}_{j}^{\mathrm{T}}+\frac{\theta}{1-\theta(m-1)}\left(\mathbf{1}_{m} \mathbf{e}_{j}^{\mathrm{T}}+\mathbf{e}_{i} \mathbf{1}_{m}^{\mathrm{T}}\right)+\frac{\theta^{2}}{(1-\theta(m-1))^{2}} \mathbf{1}_{m} \mathbf{1}_{m}^{\mathrm{T}}\right]}{\frac{1+\theta}{1-\theta(m-1)}}\right) \boldsymbol{\alpha} \\
& =\frac{2}{1+\theta}\left(\boldsymbol{\alpha}+\frac{\theta \sum_{i=1}^{i=m} \alpha_{i}}{1-\theta(m-1)} \mathbf{1}_{m}\right) \\
& -\frac{\theta[1-\theta(m-1)]}{(1+\theta)^{3}}\left[\left(\frac{\alpha_{j} \theta}{1-\theta(m-1)}+\frac{\theta^{2} \sum_{i=1}^{i=m} \alpha_{i}}{[1-\theta(m-1)]^{2}}\right) \mathbf{1}_{m}+\left(\alpha_{j}+\frac{\theta \sum_{i=1}^{i=m} \alpha_{i}}{1-\theta(m-1)}\right) \mathbf{e}_{i}\right]
\end{aligned}
$$

When $\boldsymbol{\alpha}=\alpha \mathbf{1}_{m}$, for $\alpha>0$,

$$
\begin{aligned}
& \mathbf{b}\left(g_{m}^{C}, \theta\right)+\mathbf{b}\left(g_{m}^{C}-i j, \theta\right) \\
= & \frac{2 \alpha}{1+\theta}\left(\mathbf{1}_{m}+\frac{m \theta}{1-\theta(m-1)} \mathbf{1}_{m}\right)-\frac{\alpha \theta}{(1+\theta)^{2}}\left[\frac{\theta}{1-\theta(m-1)} \mathbf{1}_{m}+\mathbf{e}_{i}\right]
\end{aligned}
$$

As a result, when $\boldsymbol{\alpha}=\alpha \mathbf{1}_{m}$, for individual $i$, the sum in terms of the unweighted Katz-Bonacich centrality is equal to: 


$$
\begin{aligned}
b_{i}\left(g_{m}^{C}, \theta\right)+b_{i}\left(g_{m}^{C}-i j, \theta\right) & =\frac{2 \alpha}{1+\theta}\left(1+\frac{m \theta}{1-\theta(m-1)}\right)-\frac{\alpha \theta}{(1+\theta)^{2}}\left[\frac{\theta}{1-\theta(m-1)}+1\right] \\
& =\frac{2 \alpha}{1+\theta}\left[\frac{1+\theta}{1-\theta(m-1)}\right]-\frac{\alpha \theta}{(1+\theta)^{2}}\left[\frac{1-\theta(m-2)}{1-\theta(m-1)}\right] \\
& =\frac{2 \alpha}{1-\theta(m-1)}-\frac{\alpha \theta}{(1+\theta)^{2}}\left[\frac{1-\theta(m-2)}{1-\theta(m-1)}\right] \\
& =\frac{\alpha\left[2+3 \theta+\theta^{2} m\right]}{(1+\theta)^{2}[1-\theta(m-1)]}
\end{aligned}
$$

Finally, the sum of the unweighted Katz-Bonacich centralities for which $\alpha=1$ for all agents is given by:

$$
b_{i}\left(g_{m}^{C}, \theta\right)+b_{i}\left(g_{m}^{C}-i j, \theta\right)=\frac{2+3 \theta+\theta^{2} m}{(1+\theta)^{2}[1-\theta(m-1)]}
$$

Therefore, the loss for individual $i$ of deleting a link $i j$ is:

$$
\begin{aligned}
B_{i}^{*}\left(g_{m}^{C}-i j\right)-B_{i}^{*}\left(g_{m}^{C}\right) & =\frac{\alpha^{2}}{2}\left[b_{i}\left(g_{m}^{C}-i j, \theta\right)-b_{i}\left(g_{m}^{C}, \theta\right)\right]\left[b_{i}\left(g_{m}^{C}-i j, \theta\right)+b_{i}\left(g_{m}^{C}, \theta\right)\right] \\
& =-\frac{\alpha^{2}}{2} \frac{\theta}{(1+\theta)^{2}}\left[\frac{1-\theta(m-2)}{1-\theta(m-1)}\right] \frac{\left[2+3 \theta+\theta^{2} m\right]}{(1+\theta)^{2}[1-\theta(m-1)]} \\
& =-\frac{\alpha^{2} \theta\left(2+3 \theta+\theta^{2} m\right)[1-\theta(m-2)]}{2(1+\theta)^{4}[1-\theta(m-1)]^{2}}
\end{aligned}
$$

What about the gain in costs?

When a type $-B$ individual deletes a link with a type $-W$, her direct gain from this deletion is (remember that $m=n^{W}+n^{B}$ ):

$$
c+\left(\frac{n^{B}-1}{n-1}\right)\left(\frac{n^{W}-1}{m-1}\right) C=c+\frac{\left(n^{B}-1\right)\left(n^{W}-1\right)}{(m-1)^{2}} C
$$

Now, when she deletes this link, her exposure rate changes and thus we need to calculate the loss in terms of costs with the remaining $n^{W}-1$ individuals of type $W$. Before the deletion of this link, the total cost of having a link with the $n^{W}-1$ individuals of type $W$ was:

$$
\left(n^{W}-1\right)\left[c+\left(\frac{n^{B}-1}{m-1}\right)\left(\frac{n^{W}-1}{m-1}\right) C\right]=\left(n^{W}-1\right)\left[c+\frac{\left(n^{B}-1\right)\left(n^{W}-1\right)}{(m-1)^{2}} C\right]
$$

After the deletion of this link, the total cost of having a link with the $n^{W}-1$ individuals of type $W$ is:

$$
\left(n^{W}-1\right)\left[c+\left(\frac{n^{B}-1}{m-2}\right)\left(\frac{n^{W}-1}{m-1}\right) C\right]=\left(n^{W}-1\right)\left[c+\frac{\left(n^{B}-1\right)\left(n^{W}-1\right)}{(m-1)(m-2)} C\right]
$$


As a result, the total gain in terms of cost for a type- $B$ individual who deletes a link with a type $-W$ is given by:

$$
\begin{aligned}
& c+\frac{\left(n^{B}-1\right)\left(n^{W}-1\right)}{(m-1)^{2}} C+\left\{\left(n^{W}-1\right)\left[c+\frac{\left(n^{B}-1\right)\left(n^{W}-1\right)}{(m-1)^{2}} C-c-\frac{\left(n^{B}-1\right)\left(n^{W}-1\right)}{(m-1)(m-2)} C\right]\right\} \\
= & c+\frac{\left(n^{B}-1\right)\left(n^{W}-1\right)}{(m-1)^{2}} C-\left\{\left(n^{W}-1\right)\left[c+\frac{\left(n^{B}-1\right)\left(n^{W}-1\right)}{(m-1)(m-2)} C-c-\frac{\left(n^{B}-1\right)\left(n^{W}-1\right)}{(m-1)^{2}} C\right]\right\} \\
= & c+\frac{\left(n^{B}-1\right)\left(n^{W}-1\right)}{(m-1)^{2}} C-\frac{\left(n^{W}-1\right)^{2}\left(n^{B}-1\right)}{(m-1)^{2}(m-2)} C \\
= & c+\left(n^{B}-1\right)\left(n^{W}-1\right)\left[\frac{n-2-n^{W}+1}{(m-1)^{2}(m-2)}\right] C \\
= & c+\frac{\left(n^{B}-1\right)^{2}\left(n^{W}-1\right)}{(m-1)^{2}(m-2)} C
\end{aligned}
$$

Since this is positive, there is a gain in terms of costs.

Proceeding in the same way, it is easily verified that the total gain in terms of cost for a type- $W$ individual who deletes a link with a type $-B$ is given by:

$$
c+\frac{\left(n^{B}-1\right)\left(n^{W}-1\right)^{2}}{(m-1)^{2}(m-2)} C
$$

Since $n^{B}<n^{W}$,

$$
c+\frac{\left(n^{B}-1\right)^{2}\left(n^{W}-1\right)}{(m-1)^{2}(m-2)} C<c+\frac{\left(n^{B}-1\right)\left(n^{W}-1\right)^{2}}{(m-1)^{2}(m-2)} C
$$

We also need to consider the gains in terms of cost when a type $-B$ individual deletes a link with another type- $B$ individual. Her direct gain from this deletion is $c$.

Now, when she deletes this link, her exposure rate changes and thus we need to calculate the loss in terms of costs with the remaining $n^{W}$ individuals of type $W$. Before the deletion of this link, the total cost of having a link with the $n^{W}$ individuals of type $W$ was:

$$
n^{W}\left[c+\left(\frac{n^{B}-1}{m-1}\right)\left(\frac{n^{W}-1}{m-1}\right) C\right]=\left(n^{W}-1\right)\left[c+\frac{\left(n^{B}-1\right)\left(n^{W}-1\right)}{(m-1)^{2}} C\right]
$$

After the deletion of this link, the total cost of having a link with the $n^{W}$ individuals of type $W$ is:

$$
n^{W}\left[c+\left(\frac{n^{B}-1}{m-2}\right)\left(\frac{n^{W}-1}{m-1}\right) C\right]=\left(n^{W}-1\right)\left[c+\frac{\left(n^{B}-1\right)\left(n^{W}-1\right)}{(m-1)(m-2)} C\right]
$$

As a result, the total gain in terms of cost for a type $-B$ individual who deletes a link with another 
type $-B$ individual is given by:

$$
\begin{gathered}
c+n^{W}\left[c+\frac{\left(n^{B}-1\right)\left(n^{W}-1\right)}{(m-1)^{2}} C-c-\frac{\left(n^{B}-1\right)\left(n^{W}-1\right)}{(m-1)(m-2)} C\right] \\
=c-n^{W}\left[c+\frac{\left(n^{B}-1\right)\left(n^{W}-1\right)}{(m-1)(m-2)} C-c-\frac{\left(n^{B}-1\right)\left(n^{W}-1\right)}{(m-1)^{2}} C\right] \\
=c-\frac{n^{W}\left(n^{B}-1\right)\left(n^{W}-1\right)}{(m-1)^{2}(m-2)} C
\end{gathered}
$$

Proceeding in the same way, it is easily verified that the total gain in terms of cost for a type- $W$ individual who deletes a link with a type $-B$ is given by:

$$
c-\frac{n^{B}\left(n^{B}-1\right)\left(n^{W}-1\right)}{(m-1)^{2}(m-2)} C
$$

We need to take the highest value between (34), (35) and (36). It is clearly:

$$
c+\frac{\left(n^{B}-1\right)\left(n^{W}-1\right)^{2}}{(m-1)^{2}(m-2)} C
$$

As a result, if

$$
c+\frac{\left(n^{B}-1\right)\left(n^{W}-1\right)^{2}}{(m-1)^{2}(m-2)} C<\frac{\alpha^{2} \theta\left(2+3 \theta+\theta^{2} m\right)[1-\theta(m-2)]}{2(1+\theta)^{4}[1-\theta(m-1)]^{2}}
$$

then nobody in the network (type $W$ or type $B$ ) will want to delete a link and the complete network with $m$ individuals is pairwise stable. This is condition (23).

(ii) We want now to show that the network for which each community is separated and each community forms a complete network, i.e. each agent of type $W$ has $n^{W}-1$ links and each agent of type $B$ has $n^{B}-1$ links, is pairwise stable.

First, we need to check that nobody wants to delete a link with someone from the same community. Proceeding exactly as in $(i)$, given that $n^{B}<n^{W}$, the condition for which nobody wants to severe a link is:

$$
c<\frac{\alpha^{2} \theta\left(2+3 \theta+\theta^{2} n^{B}\right)\left[1-\theta\left(n^{B}-2\right)\right]}{2(1+\theta)^{4}\left[1-\theta\left(n^{B}-1\right)\right]^{2}}
$$

and

$$
c<\frac{\alpha^{2} \theta\left(2+3 \theta+\theta^{2} n^{W}\right)\left[1-\theta\left(n^{W}-2\right)\right]}{2(1+\theta)^{4}\left[1-\theta\left(n^{W}-1\right)\right]^{2}}
$$

It is easily verified that

$$
\frac{\alpha^{2} \theta\left(2+3 \theta+\theta^{2} m\right)[1-\theta(m-2)]}{2(1+\theta)^{4}[1-\theta(m-1)]^{2}}
$$


is increasing in $m$. As a result, since $n^{B}<n^{W}$,

$$
\frac{\alpha^{2} \theta\left(2+3 \theta+\theta^{2} n^{B}\right)\left[1-\theta\left(n^{B}-2\right)\right]}{2(1+\theta)^{4}\left[1-\theta\left(n^{B}-1\right)\right]^{2}}<\frac{\alpha^{2} \theta\left(2+3 \theta+\theta^{2} n^{W}\right)\left[1-\theta\left(n^{W}-2\right)\right]}{2(1+\theta)^{4}\left[1-\theta\left(n^{W}-1\right)\right]^{2}}
$$

Thus, if

$$
c<\frac{\alpha^{2} \theta\left(2+3 \theta+\theta^{2} n^{B}\right)\left[1-\theta\left(n^{B}-2\right)\right]}{2(1+\theta)^{4}\left[1-\theta\left(n^{B}-1\right)\right]^{2}}
$$

then nobody in her own network (type $W$ or type $B$ ) will want to delete a link with someone from the same community.

Now, we need to check for link-formation deviation between the two communities.

\section{Connecting two complete components}

Let $q>1$ and $n>1$. Assume that

$$
\mathbf{G}_{q n}^{C}=\left(\begin{array}{cc}
\mathbf{1}_{q} \mathbf{1}_{q}^{\mathrm{T}}-\mathbf{I}_{q} & \mathbf{0}_{q} \mathbf{0}_{n}^{\mathrm{T}} \\
\mathbf{0}_{n} \mathbf{0}_{q}^{\mathrm{T}} & \mathbf{1}_{n} \mathbf{1}_{n}^{\mathrm{T}}-\mathbf{I}_{n}
\end{array}\right)
$$

where $\mathbf{0}_{q}$ is the $q$-vector of zeros and $\mathbf{G}_{q n}^{C}$ is a $(q+n) \times(q+n)$ matrix. Observe that $\mathbf{G}_{q n}$, a block-diagonal matrix, is the adjacency matrix of two complete separated networks, one with all individuals of type $W\left(q=n^{W}\right)$ and one with all individuals of type $B\left(n=n^{B}\right)$ and with no connection between the two networks.

Observe that $m=q+n$. Then, we would like to calculate $\mathbf{b}\left(g_{q n}^{C}, \theta\right)=\left(\mathbf{I}_{m}-\theta \mathbf{G}_{q n}^{C}\right)^{-1}$, the Katz-Bonacich centrality of each agent in each network. We have:

$$
\begin{aligned}
& \left(\mathbf{I}_{m}-\theta \mathbf{G}_{q n}^{C}\right)^{-1} \\
= & \left(\begin{array}{cc}
(1+\theta) \mathbf{I}_{q}-\theta \mathbf{1}_{q} \mathbf{1}_{q}^{\mathrm{T}} & \mathbf{0}_{q} \mathbf{0}_{n}^{\mathrm{T}} \\
\mathbf{0}_{n} \mathbf{0}_{q}^{\mathrm{T}} & (1+\theta) \mathbf{I}_{m}-\theta \mathbf{1}_{n} \mathbf{1}_{n}^{\mathrm{T}}
\end{array}\right)^{-1} \\
= & \left(\begin{array}{cc}
\left.(1+\theta) \mathbf{I}_{q}-\theta \mathbf{1}_{q} \mathbf{1}_{q}^{\mathrm{T}}\right)^{-1} & \mathbf{0}_{q} \mathbf{0}_{n}^{\mathrm{T}} \\
\mathbf{0}_{n} \mathbf{0}_{q}^{\mathrm{T}} & \left((1+\theta) \mathbf{I}_{m}-\theta \mathbf{1}_{n} \mathbf{1}_{n}^{\mathrm{T}}\right)^{-1}
\end{array}\right) \\
= & \left(\begin{array}{cc}
\frac{1}{1+\theta}\left(\mathbf{I}_{q}-\frac{\theta}{1-\theta(q-1)} \mathbf{1}_{q} \mathbf{1}_{q}^{\mathrm{T}}\right) & \mathbf{0}_{q} \mathbf{0}_{n}^{\mathrm{T}} \\
\mathbf{0}_{n} \mathbf{0}_{q}^{\mathrm{T}} & \frac{1}{1+\theta}\left(\mathbf{I}_{m}-\frac{\theta}{1-\theta(n-1)} \mathbf{1}_{n} \mathbf{1}_{n}^{\mathrm{T}}\right)
\end{array}\right) \\
= & \frac{1}{1+\theta} \mathbf{I}_{m}+\frac{\theta}{1+\theta}\left(\begin{array}{cc}
\frac{1}{1-\theta(q-1)} \mathbf{1}_{q} \mathbf{1}_{q}^{\mathrm{T}} & \mathbf{0}_{q} \mathbf{0}_{n}^{\mathrm{T}} \\
\mathbf{0}_{n} \mathbf{0}_{q}^{\mathrm{T}} & \frac{\theta}{1-\theta(n-1)} \mathbf{1}_{n} \mathbf{1}_{n}^{\mathrm{T}}
\end{array}\right)
\end{aligned}
$$


Now consider adding a (bridge) link to the network $\mathbf{G}_{q n}^{C}$ between an individual from network $q$ (type $W$ ), say individual 1 , and an individual from network $m$ (type $B$ ), say individual $q+n=m$, which results in a new network: $\mathbf{G}_{q n}^{C}+\mathbf{e}_{1} \mathbf{e}_{m}^{\mathrm{T}}$. We have:

$$
\begin{aligned}
& \left(\mathbf{I}_{m}-\theta\left(\mathbf{G}_{q n}^{C}+\mathbf{e}_{1} \mathbf{e}_{m}^{\mathrm{T}}\right)\right)^{-1} \\
= & \left(\left(\mathbf{I}_{m}-\theta \mathbf{G}_{q n}^{C}\right)-\theta \mathbf{e}_{1} \mathbf{e}_{m}^{\mathrm{T}}\right)^{-1} \\
= & \left(\mathbf{I}_{m}-\theta \mathbf{G}_{q n}^{C}\right)^{-1}+\frac{\theta\left(\mathbf{I}_{m}-\theta \mathbf{G}_{q n}^{C}\right)^{-1} \mathbf{e}_{1} \mathbf{e}_{m}^{\mathrm{T}}\left(\mathbf{I}_{m}-\theta \mathbf{G}_{q n}^{C}\right)^{-1}}{1-\theta \mathbf{e}_{m}^{\mathrm{T}}\left(\mathbf{I}_{m}-\theta \mathbf{G}_{q n}^{C}\right)^{-1} \mathbf{e}_{1}}
\end{aligned}
$$

where

$$
\begin{aligned}
& \theta\left(\mathbf{I}_{m}-\theta \mathbf{G}_{q n}^{C}\right)^{-1} \mathbf{e}_{1} \mathbf{e}_{m}^{\mathrm{T}}\left(\mathbf{I}_{m}-\theta \mathbf{G}_{q n}^{C}\right)^{-1} \\
= & {\left[\frac{1}{1+\theta} \mathbf{I}_{m}+\frac{\theta}{1+\theta}\left(\begin{array}{cc}
\frac{1}{1-\theta(q-1)} \mathbf{1}_{q} \mathbf{1}_{q}^{\mathrm{T}} & \mathbf{0}_{q} \mathbf{0}_{n}^{\mathrm{T}} \\
\mathbf{0}_{n} \mathbf{0}_{q}^{\mathrm{T}} & \frac{\theta}{1-\theta(n-1)} \mathbf{1}_{n} \mathbf{1}_{n}^{\mathrm{T}}
\end{array}\right)\right] \mathbf{e}_{1} } \\
& \times \mathbf{e}_{m}^{\mathrm{T}}\left[\frac{1}{1+\theta} \mathbf{I}_{m}+\frac{\theta}{1+\theta}\left(\begin{array}{cc}
\frac{1}{1-\theta(q-1)} \mathbf{1}_{q} \mathbf{1}_{q}^{\mathrm{T}} & \mathbf{0}_{q} \mathbf{0}_{n}^{\mathrm{T}} \\
\mathbf{0}_{n} \mathbf{0}_{q}^{\mathrm{T}} & \frac{\theta}{1-\theta(n-1)} \mathbf{1}_{n} \mathbf{1}_{n}^{\mathrm{T}}
\end{array}\right)\right] \\
= & {\left[\left(\frac{1}{1+\theta}\right) \mathbf{e}_{1}+\frac{\theta}{1+\theta}\left(\begin{array}{c}
\frac{1}{1-\theta(q-1)} \mathbf{1}_{q} \\
\mathbf{0}_{n}
\end{array}\right)\right]\left[\left(\frac{1}{1+\theta}\right) \mathbf{e}_{m}^{\mathrm{T}}+\frac{\theta}{1+\theta}\left(\begin{array}{c}
\frac{1}{1-\theta(n-1)} \mathbf{1}_{n}^{\mathrm{T}} \\
\mathbf{0}_{q}^{\mathrm{T}}
\end{array}\right)\right] } \\
= & \frac{1}{(1+\theta)^{2}} \mathbf{e}_{1} \mathbf{e}_{m}^{\mathrm{T}}+\frac{\theta}{(1+\theta)^{2}} \mathbf{e}_{1}\left(\begin{array}{c}
\frac{1}{1-\theta(n-1)} \mathbf{1}_{n}
\end{array}\right)^{\mathrm{0}}+\frac{\theta}{(1+\theta)^{2}}\left(\begin{array}{c}
\frac{1}{1-\theta(q-1)} \mathbf{1}_{q} \\
\mathbf{0}_{n}
\end{array}\right) \mathbf{e}_{m}^{\mathrm{T}} \\
& +\frac{\theta^{2}}{(1+\theta)^{2}}\left(\begin{array}{c}
\frac{1}{1-\theta(q-1)} \mathbf{1}_{q} \\
\mathbf{0}_{n}
\end{array}\right)\left(\begin{array}{c}
\mathbf{0}_{q} \\
\frac{1}{1-\theta(n-1)} \mathbf{1}_{n}
\end{array}\right)^{\mathrm{T}}
\end{aligned}
$$

and where

$$
\begin{aligned}
& 1-\theta \mathbf{e}_{m}^{\mathrm{T}}\left(\mathbf{I}_{m}-\theta \mathbf{G}_{q n}^{C}\right)^{-1} \mathbf{e}_{1} \\
= & 1-\theta \mathbf{e}_{m}^{\mathrm{T}}\left[\frac{1}{1+\theta} \mathbf{I}_{m}+\frac{\theta}{1+\theta}\left(\begin{array}{cc}
\frac{1}{1-\theta(q-1)} \mathbf{1}_{q} \mathbf{1}_{q}^{\mathrm{T}} & \mathbf{0}_{q} \mathbf{0}_{n}^{\mathrm{T}} \\
\mathbf{0}_{n} \mathbf{0}_{q}^{\mathrm{T}} & \frac{\theta}{1-\theta(n-1)} \mathbf{1}_{n} \mathbf{1}_{n}^{\mathrm{T}}
\end{array}\right)\right] \mathbf{e}_{1} \\
= & 1-\theta \mathbf{e}_{m}^{\mathrm{T}}\left[\frac{1}{1+\theta} \mathbf{e}_{1}+\frac{\theta}{1+\theta}\left(\begin{array}{c}
\frac{1}{1-\theta(q-1)} \mathbf{1}_{q} \\
\mathbf{0}_{n}
\end{array}\right)\right] \\
= & 1
\end{aligned}
$$

Assume that $\alpha=1$ for all agents. The block-diagonal structure of $\mathbf{G}_{q n}^{C}$ implies that the unweighted Katz-Bonacih centrality is equal to (see also Lemma 1)):

$$
\mathbf{b}\left(g_{q n}^{C}, \theta\right)=\left(\begin{array}{c}
\frac{1}{1-\theta(q-1)} \mathbf{1}_{q} \\
\frac{1}{1-\theta(n-1)} \mathbf{1}_{n}
\end{array}\right)
$$


We find that:

$$
\begin{aligned}
& \mathbf{b}\left(g_{q n}^{C}+\mathbf{e}_{1} \mathbf{e}_{m}^{\mathrm{T}}, \theta\right) \\
& =\left(\mathbf{I}_{m}-\theta\left(\mathbf{G}_{q n}^{C}+\mathbf{e}_{1} \mathbf{e}_{m}^{\mathrm{T}}\right)\right)^{-1} \mathbf{1}_{m} \\
& =\left(\mathbf{I}_{m}-\theta \mathbf{G}_{q n}^{C}\right)^{-1} \mathbf{1}_{m} \\
& +\theta\left[\begin{array}{c}
\frac{1}{(1+\theta)^{2}} \mathbf{e}_{1} \mathbf{e}_{m}^{\mathrm{T}}+\frac{\theta}{(1+\theta)^{2}} \mathbf{e}_{1}\left(\begin{array}{c}
\mathbf{0}_{q} \\
\frac{1}{1-\theta(n-1)} \mathbf{1}_{n}
\end{array}\right)^{\mathrm{T}}+\frac{\theta}{(1+\theta)^{2}}\left(\begin{array}{c}
\frac{1}{1-\theta(q-1)} \mathbf{1}_{q} \\
\mathbf{0}_{n}
\end{array}\right) \mathbf{e}_{m}^{\mathrm{T}} \\
+\frac{\theta^{2}}{(1+\theta)^{2}}\left(\begin{array}{c}
\frac{1}{1-\theta(q-1)} \mathbf{1}_{q} \\
\mathbf{0}_{n}
\end{array}\right)\left(\begin{array}{c}
\mathbf{0}_{q} \\
\frac{1}{1-\theta(n-1)} \mathbf{1}_{n}
\end{array}\right)^{\mathrm{T}}
\end{array}\right] \mathbf{1}_{m} \\
& =\mathbf{b}\left(g_{q n}^{C}, \theta\right)+\frac{\theta}{(1+\theta)^{2}} \mathbf{e}_{1}+\frac{n \theta^{2}}{(1+\theta)^{2}[1-\theta(n-1)]} \mathbf{e}_{1}+\frac{\theta^{2}}{(1+\theta)^{2}}\left(\begin{array}{c}
\frac{1}{1-\theta(q-1)} \mathbf{1}_{q} \\
\mathbf{0}_{n}
\end{array}\right) \\
& +\frac{n \theta^{3}}{(1+\theta)^{2}[1-\theta(n-1)]}\left(\begin{array}{c}
\frac{1}{1-\theta(q-1)} \mathbf{1}_{q} \\
\mathbf{0}_{n}
\end{array}\right) \\
& =\mathbf{b}\left(g_{q n}^{C}, \theta\right)+\frac{\theta}{(1+\theta)[1-\theta(n-1)]} \mathbf{e}_{1}+\frac{\theta^{2}}{(1+\theta)[1-\theta(n-1)]}\left(\begin{array}{c}
\frac{1}{1-\theta(q-1)} \mathbf{1}_{q} \\
\mathbf{0}_{n}
\end{array}\right)
\end{aligned}
$$

Denote the new link between 1 and $m$ by $1 m$. Then, the change in Katz-Bonacich centrality by adding the directed link $1 m$ to $\mathbf{G}_{q n}^{C}$ is given by:

$$
\begin{aligned}
& \mathbf{b}\left(g_{q n}^{C}+1 m, \theta\right)-\mathbf{b}\left(g_{q n}^{C}, \theta\right) \\
= & \frac{\theta}{(1+\theta)[1-\theta(n-1)]} \mathbf{e}_{1}+\frac{\theta^{2}}{(1+\theta)[1-\theta(n-1)]}\left(\begin{array}{c}
\frac{1}{1-\theta(q-1)} \mathbf{1}_{q} \\
\mathbf{0}_{n}
\end{array}\right)
\end{aligned}
$$

As a result, for individual 1, we have

$$
\begin{aligned}
& b_{1}\left(g_{q n}^{C}+1 m, \theta\right)-b_{1}\left(g_{q n}^{C}, \theta\right) \\
= & \frac{\theta}{(1+\theta)[1-\theta(n-1)]}+\frac{\theta^{2}}{(1+\theta)[1-\theta(n-1)]} \frac{1}{1-\theta(q-1)} \\
= & \frac{\theta[1-\theta(q-1)]+\theta^{2}}{(1+\theta)[1-\theta(n-1)][1-\theta(q-1)]}
\end{aligned}
$$

Similarly, we can calculate:

$$
\begin{aligned}
& \mathbf{b}\left(g_{q n}^{C}+\mathbf{e}_{1} \mathbf{e}_{m}^{\mathrm{T}}, \theta\right)+\mathbf{b}\left(g_{q n}^{C}, \theta\right) \\
= & 2 \mathbf{b}\left(g_{q n}^{C}, \theta\right)+\frac{\theta}{(1+\theta)[1-\theta(n-1)]} \mathbf{e}_{1}+\frac{\theta^{2}}{(1+\theta)[1-\theta(n-1)]}\left(\begin{array}{c}
\frac{1}{1-\theta(q-1)} \mathbf{1}_{q} \\
\mathbf{0}_{n}
\end{array}\right) \\
= & 2\left(\begin{array}{c}
\frac{1}{1-\theta(q-1)} \mathbf{1}_{q} \\
\frac{1}{1-\theta(n-1)} \mathbf{1}_{n}
\end{array}\right)+\frac{\theta}{(1+\theta)[1-\theta(n-1)]} \mathbf{e}_{1}+\frac{\theta^{2}}{(1+\theta)[1-\theta(n-1)]}\left(\begin{array}{c}
\frac{1}{1-\theta(q-1)} \mathbf{1}_{q} \\
\mathbf{0}_{n}
\end{array}\right)
\end{aligned}
$$


As a result, for individual 1:

$$
\begin{aligned}
& b_{1}\left(g_{q n}^{C}+1 m, \theta\right)+b_{1}\left(g_{q n}^{C}, \theta\right) \\
= & \frac{2}{1-\theta(q-1)}+\frac{\theta[1-\theta(q-1)]+\theta^{2}}{(1+\theta)[1-\theta(n-1)][1-\theta(q-1)]}
\end{aligned}
$$

Therefore, the gain for individual 1 of adding a bridge link $1 m$ is:

$$
\begin{aligned}
& B_{1}^{*}\left(g_{q n}^{C}+1 m\right)-B_{1}^{*}\left(g_{q n}^{C}\right) \\
= & \frac{\alpha^{2}}{2}\left[b_{1}\left(g_{q n}^{C}+1 m, \theta\right)\right]^{2}-\frac{1}{2}\left[b_{1}\left(g_{q n}^{C}, \theta\right)\right]^{2} \\
= & \frac{\alpha^{2}}{2}\left[b_{1}\left(g_{q n}^{C}+1 m, \theta\right)-b_{1}\left(g_{q n}^{C}, \theta\right)\right]\left[b_{1}\left(g_{q n}^{C}+1 m, \theta\right)+b_{1}\left(g_{q n}^{C}, \theta\right)\right] \\
= & \frac{\alpha^{2}}{2}\left[\frac{\theta[1-\theta(q-1)]+\theta^{2}}{(1+\theta)[1-\theta(n-1)][1-\theta(q-1)]}\right]\left[\frac{2}{1-\theta(q-1)}+\frac{\theta[1-\theta(q-1)]+\theta^{2}}{(1+\theta)[1-\theta(n-1)][1-\theta(q-1)]}\right]
\end{aligned}
$$

Since $q=n^{W}$ and $n=n^{B}$, we have:

$$
\begin{aligned}
& B_{1}^{*}\left(g_{n^{W} n^{B}}^{C}+1 m\right)-B_{1}^{*}\left(g_{n^{W} n^{B}}^{C}\right) \\
= & \frac{\alpha^{2}\left(\theta\left[1-\theta\left(n^{W}-1\right)\right]+\theta^{2}\right)\left(2(1+\theta)\left[1-\theta\left(n^{B}-1\right)\right]+\theta\left[1-\theta\left(n^{W}-1\right)+\theta\right]\right)}{2(1+\theta)^{2}\left[1-\theta\left(n^{B}-1\right)\right]^{2}\left[1-\theta\left(n^{W}-1\right)\right]^{2}}
\end{aligned}
$$

As a result, individual 1 will not form a bridge link between individual 1 (from the type- $W$ network) and individual $m$ if and only if

$$
B_{1}^{*}\left(g_{n^{W} n^{B}}^{C}+1 m\right)-B_{1}^{*}\left(g_{n^{W} n^{B}}^{C}\right)<c+\frac{\left(n^{B}-1\right)\left(n^{W}-1\right)}{n^{B} n^{W}} C
$$

which is equivalent to:

$$
\begin{aligned}
& \frac{\alpha^{2}\left(\theta\left[1-\phi\left(n^{W}-1\right)\right]+\theta^{2}\right)\left(2(1+\theta)\left[1-\phi\left(n^{B}-1\right)\right]+\theta\left[1-\phi\left(n^{W}-1\right)+\theta\right]\right)}{2(1+\theta)^{2}\left[1-\phi\left(n^{B}-1\right)\right]^{2}\left[1-\phi\left(n^{W}-1\right)\right]^{2}} \\
< & c+\frac{\left(n^{B}-1\right)\left(n^{W}-1\right)}{n^{B} n^{W}} C
\end{aligned}
$$

Thus if (37) and (38) hold, then the separated network is pairwise stable. 


\section{Proof of Proposition 13}

Let us denote by $B_{i}^{*}(g)$, the positive part (benefits) of the equilibrium utility function (20), i.e. $B_{i}^{*}(g) \equiv \frac{\alpha^{2}}{2}\left[b_{i}(g, \theta)\right]^{2}$.

Let us show that the network described in Figure 1 is an equilibrium network.

Let us start with link deletion:

- Any minority agent (individual 2 or 3 ) has no incentives to sever a link with individual 1 (type $W)$ if

$$
c<\frac{\alpha^{2}}{2}\left[\left(\frac{1+\theta}{1-2 \theta^{2}}\right)^{2}-1\right]
$$

Indeed, the utility of individual 2 (or 3 ) before the link is severed is:

$$
U_{2}^{*}\left(g^{S}\right)=\frac{\alpha^{2}}{2}\left[b_{2}\left(g^{S}, \theta\right)\right]^{2}-c_{21}\left(g^{S}\right)
$$

As a result, if $\theta<1 / \sqrt{2}$, we have:

$$
\left(\begin{array}{c}
b_{1}\left(g^{S}, \theta\right) \\
b_{2}\left(g^{S}, \theta\right) \\
b_{3}\left(g^{S}, \theta\right)
\end{array}\right)=\frac{1}{1-2 \theta^{2}}\left(\begin{array}{c}
1+2 \theta \\
1+\theta \\
1+\theta
\end{array}\right)
$$

and thus, ${ }^{17}$ if $\theta<1 / \sqrt{2}$,

$$
B_{2}^{*}\left(g^{I}\right)-B_{2}^{*}\left(g^{S}\right)=\frac{\alpha^{2}}{2}\left[1-\left(\frac{1+\theta}{1-2 \theta^{2}}\right)^{2}\right]=-\frac{\alpha^{2}}{2} \frac{\theta\left(2+5 \theta-4 \theta^{3}\right)}{\left(1-2 \theta^{2}\right)^{2}}<0
$$

Let is now determine the gains in terms of costs. It is equal to: $c$, which is the cost for individual 2 or 3 of having a link with individual 1. As a result, if

$$
\frac{\alpha^{2}}{2}\left[1-\left(\frac{1+\theta}{1-2 \theta^{2}}\right)^{2}\right]+c<0
$$

then individual 2 or 3 will never delete a link with individual 1. This is condition (39).

- The majority agent (individual 1) will not sever a link with a minority agent (individual 2 or 3) if

$$
c<\frac{\alpha^{2}}{2}\left[\left(\frac{1+\theta}{1-2 \theta^{2}}\right)^{2}-\frac{1}{(1-\theta)^{2}}\right]
$$

Let us first calculate the loss of deleting such a link. We have seen that, before deleting this link, if $\theta<1 / \sqrt{2}$, we have:

$$
\left(\begin{array}{c}
b_{1}\left(g^{S}, \theta\right) \\
b_{2}\left(g^{S}, \theta\right) \\
b_{3}\left(g^{S}, \theta\right)
\end{array}\right)=\frac{1}{1-2 \theta^{2}}\left(\begin{array}{c}
1+2 \theta \\
1+\theta \\
1+\theta
\end{array}\right)
$$

\footnotetext{
${ }^{17}$ The superscript $S$ denotes the star network while the superscript $I$ denotes the isolated (empty) network.
} 
After deleting the link (for example link 12), the network is a dyad with one link 13, and, if $\theta<1$,

$$
\left(\begin{array}{l}
b_{1}\left(g^{S}, \theta\right) \\
b_{2}\left(g^{S}, \theta\right)
\end{array}\right)=\frac{1}{1-\theta}\left(\begin{array}{l}
1 \\
1
\end{array}\right)
$$

As a result, if $\theta<1 / \sqrt{2}$, then ${ }^{18}$

$$
B_{1}^{*}\left(g^{D}\right)-B_{1}^{*}\left(g^{S}\right)=\frac{\alpha^{2}}{2}\left[\frac{1}{(1-\theta)^{2}}-\left(\frac{1+\theta}{1-2 \theta^{2}}\right)^{2}\right]=-\frac{\theta^{2}\left(2-3 \theta^{2}\right)}{\left(1-\theta-2 \theta^{2}+2 \theta^{3}\right)^{2}}<0
$$

Let is now determine the gains in terms of costs. It is equal to: $2 c-c=c$. As a result, if

$$
\frac{\alpha^{2}}{2}\left[\frac{1}{(1-\theta)^{2}}-\left(\frac{1+\theta}{1-2 \theta^{2}}\right)^{2}\right]+c<0
$$

then individual 1 will never delete a link with individual 2 or 3 . This is condition (40).

It is easily verified that, if $\theta<1 / \sqrt{2}$, conditions (39) and (40) reduce to:

$$
c<\frac{\alpha^{2}}{2}\left[\left(\frac{1+\theta}{1-2 \theta^{2}}\right)^{2}-\frac{1}{(1-\theta)^{2}}\right]
$$

since

$$
\frac{\alpha^{2}}{2}\left[\left(\frac{1+\theta}{1-2 \theta^{2}}\right)^{2}-\frac{1}{(1-\theta)^{2}}\right]<\frac{\alpha^{2}}{2}\left[\left(\frac{1+\theta}{1-2 \theta^{2}}\right)^{2}-1\right]
$$

Let us now consider link creation:

- The majority agent (individual 1) cannot create more links since she is already linked to all agents in the network.

- The minority agent (individual 2) will not create a link with the minority agent 3 if

$$
c>\frac{\alpha^{2}}{2}\left[\frac{1}{(1-2 \theta)^{2}}-\left(\frac{1+\theta}{1-2 \theta^{2}}\right)^{2}\right]
$$

We have seen that, before the creation of the link 23 , if $\theta<1 / \sqrt{2}$, we have:

$$
\left(\begin{array}{c}
b_{1}\left(g^{S}, \theta\right) \\
b_{2}\left(g^{S}, \theta\right) \\
b_{3}\left(g^{S}, \theta\right)
\end{array}\right)=\frac{1}{1-2 \theta^{2}}\left(\begin{array}{c}
2 \theta+1 \\
\theta+1 \\
\theta+1
\end{array}\right)
$$

If the link 23 is created so that the network is complete, then, if $\theta<1 / 2$,

$$
\left(\begin{array}{c}
b_{1}\left(g^{C}, \theta\right) \\
b_{2}\left(g^{C}, \theta\right) \\
b_{3}\left(g^{C}, \theta\right)
\end{array}\right)=\frac{1}{1-2 \theta}\left(\begin{array}{l}
1 \\
1 \\
1
\end{array}\right)
$$

\footnotetext{
${ }^{18}$ The superscript $D$ denotes the dyad network, i.e. the complete network with two agents.
} 
As a result, if $\theta<1 / 2$, the gain of creating the link 23 is equal to (for agent 2 or 3 ):

$$
B_{2}^{*}\left(g^{C}\right)-B_{2}^{*}\left(g^{S}\right)=\frac{\alpha^{2}}{2}\left[\frac{1}{(1-2 \theta)^{2}}-\left(\frac{1+\theta}{1-2 \theta^{2}}\right)^{2}\right]=\frac{\alpha^{2}}{2} \frac{\theta\left(2-\theta-4 \theta^{2}\right)}{\left(1-2 \theta-2 \theta^{2}+4 \theta^{3}\right)^{2}}>0
$$

When the link 23 is created, the cost increases by $c$ since the total cost for individual 3 before creating the link 23 is: $c$ while the total cost for individual 3 after creating the link 23 is: $2 c$. As a result, individual 2 will never create a link with individual 3 if

$$
\frac{\alpha^{2}}{2}\left[\frac{1}{1-2 \theta}-\frac{1+\theta}{1-2 \theta^{2}}\right]-c<0
$$

This is condition (42).

We are thus left with two conditions (41) and (42). Combining them gives

$$
\frac{1}{(1-2 \theta)^{2}}-\left(\frac{1+\theta}{1-2 \theta^{2}}\right)^{2}<\frac{2 c}{\alpha^{2}}<\left(\frac{1+\theta}{1-2 \theta^{2}}\right)^{2}-\frac{1}{(1-\theta)^{2}}
$$

If $\theta<1 / \sqrt{2}$, it is easily verified that

$$
\frac{1}{(1-2 \theta)^{2}}-\left(\frac{1+\theta}{1-2 \theta^{2}}\right)^{2}<\left(\frac{1+\theta}{1-2 \theta^{2}}\right)^{2}-\frac{1}{(1-\theta)^{2}}
$$

By simplifying (43), we obtain condition (26).

Proof of Proposition 14: Let us denote by $B_{i}^{*}(g)$, the positive part (benefits) of the equilibrium utility function $(20)$, i.e. $B_{i}^{*}(g) \equiv \frac{\alpha^{2}}{2}\left[b_{i}(g, \theta)\right]^{2}$. Since we have a complete network, we can only consider the deviations due to link deletion and not link creation (nobody can form new links). Let us show that the network described in Figure 2 is an equilibrium network.

- Any minority agent (take individual 2 without loss of generality) has no incentive to sever a link with individual 2 (type $B$ ) if

$$
c<\frac{\alpha^{2}}{2}\left[\frac{1}{(1-2 \theta)^{2}}-\left(\frac{1+\theta}{1-2 \theta^{2}}\right)^{2}\right]
$$

Indeed, before the link 23 is severed, the network is complete, and, if $\theta<1 / 2$,

$$
\left(\begin{array}{c}
b_{1}\left(g^{C}, \theta\right) \\
b_{2}\left(g^{C}, \theta\right) \\
b_{3}\left(g^{C}, \theta\right)
\end{array}\right)=\frac{1}{1-2 \theta}\left(\begin{array}{l}
1 \\
1 \\
1
\end{array}\right)
$$

When the link 23 is severed, the network is a star, and, if $\theta<1 / \sqrt{2}$, we have:

$$
\left(\begin{array}{l}
b_{1}\left(g^{S}, \theta\right) \\
b_{2}\left(g^{S}, \theta\right) \\
b_{3}\left(g^{S}, \theta\right)
\end{array}\right)=\frac{1}{1-2 \theta^{2}}\left(\begin{array}{c}
1+2 \theta \\
1+\theta \\
1+\theta
\end{array}\right)
$$


Thus, if $\theta<1 / \sqrt{2}$,

$$
B_{2}^{*}\left(g^{S}\right)-B_{2}^{*}\left(g^{C}\right)=\frac{\alpha^{2}}{2}\left[\left(\frac{1+\theta}{1-2 \theta^{2}}\right)^{2}-\frac{1}{(1-2 \theta)^{2}}\right]=-\frac{\theta\left(2-\theta-4 \theta^{2}\right)}{\left(1-2 \theta-2 \theta^{2}+4 \theta^{3}\right)^{2}}<0
$$

Let is now determine the gains in terms of costs. It is clearly equal to $c$. As a result, if

$$
\frac{\alpha^{2}}{2}\left[\left(\frac{1+\theta}{1-2 \theta^{2}}\right)^{2}-\frac{1}{(1-2 \theta)^{2}}\right]+c<0
$$

then individual 2 or 3 will never delete a link with individual 1. This is condition (44).

- Any minority agent (take individual 2 without loss of generality) has no incentive to sever a link with individual 1 (type $W$ ) if condition (44) holds. Indeed, before the link 12 is severed, the network is complete, and, if $\theta<1 / 2$,

$$
\left(\begin{array}{c}
b_{1}\left(g^{C}, \theta\right) \\
b_{2}\left(g^{C}, \theta\right) \\
b_{3}\left(g^{C}, \theta\right)
\end{array}\right)=\frac{1}{1-2 \theta}\left(\begin{array}{l}
1 \\
1 \\
1
\end{array}\right)
$$

When the link 12 is severed, we have star but it is individual 3 who is the star. If $\theta<1 / \sqrt{2}$,

$$
\left(\begin{array}{c}
b_{1}^{S}(g, \theta) \\
b_{2}^{S}(g, \theta) \\
b_{3}^{S}(g, \theta)
\end{array}\right)=\frac{1}{1-2 \theta^{2}}\left(\begin{array}{c}
1+\theta \\
1+\theta \\
1+2 \theta
\end{array}\right)
$$

Therefore,

$$
B_{2}^{*}\left(g^{S}\right)-B_{2}^{*}\left(g^{C}\right)=\frac{\alpha^{2}}{2}\left[\left(\frac{1+\theta}{1-2 \theta^{2}}\right)^{2}-\frac{1}{(1-2 \theta)^{2}}\right]=-\frac{\theta\left(2-\theta-4 \theta^{2}\right)}{\left(1-2 \theta-2 \theta^{2}+4 \theta^{3}\right)^{2}}<0
$$

Let is now determine the gains in terms of costs. It is clearly equal to $c$. As a result, if

$$
\frac{\alpha^{2}}{2}\left[\left(\frac{1+\theta}{1-2 \theta^{2}}\right)^{2}-\frac{1}{(1-2 \theta)^{2}}\right]+c<0
$$

then individual 2 or 3 will never delete a link with individual 1. This is condition (44).

- The majority agent (individual 1) will not sever a link with a minority agent (individual 2 or 3 ) if condition (44) holds. The intuition is as before since, when the link 12 is severed, there is a star network where individual 1 is a peripheral agent. So the loss is as before and the gain is still $c$.

Thus condition (44) guarantees that the complete network of Figure 2 is pairwise stable. By developing (44), we obtain (27) 